\title{
Emergent non-Fermi-liquid phenomena in multipolar quantum impurity systems
}

\author{
Adarsh S. Patri ${ }^{0}$, Ilia Khait, and Yong Baek Kim \\ Department of Physics and Centre for Quantum Materials, University of Toronto, Toronto, Ontario, Canada M5S 1A7
}

(Received 6 November 2019; revised manuscript received 7 February 2020; accepted 4 February 2020; published 4 March 2020)

\begin{abstract}
Discovery of novel spin-orbital entangled quantum ground states paves an important avenue for controllable quantum materials via unique couplings to the lattice and other external perturbations. In this work, motivated by recent experiments on cubic heavy fermion materials with multipolar local moments, we theoretically investigate strongly interacting spin-orbital entangled quantum ground states in multipolar quantum impurity systems. Here itinerant electrons are interacting with the local moments carrying quadrupolar and octupolar moments, in contrast to the conventional Kondo problem with dipolar local moments. Using perturbative renormalization group methods, we uncover a number of non-Fermi-liquid ground states, which are characterized by an absence of well-defined quasiparticles and singular power-law behaviors in physical properties. We show that the non-Fermi-liquid states found here are outside the known categories of non-Fermi-liquid states in the conventional multi-channel Kondo problem. This work lays a novel ground for the identification of unexpected non-Fermi-liquid phases in many strongly spin-orbital coupled quantum materials.
\end{abstract}

DOI: 10.1103/PhysRevResearch.2.013257

\section{INTRODUCTION}

Understanding non-Fermi-liquid states may be the key to explaining unconventional superconductivity and other broken symmetry states in strongly correlated itinerant electron systems [1-6]. This is in contrast to the Fermi liquid states with well-defined quasiparticles, which is the basis for our understanding of weakly correlated electron systems. In the traditional picture of heavy fermion materials, the RKKY interaction [7] between dipolar local moments (or spins) competes with the formation of a Fermi liquid state with a large Fermi surface [8,9], facilitated by the Kondo coupling between the dipolar local moment and conduction electrons. This competition leads to the emergence of non-Fermi liquid behaviors, which (as seen in various experiments $[10,11]$ ) are often attributed to quantum critical phenomena associated with such quantum phase transitions.

Such a simple picture, however, may change drastically if the local moments are higher-order multipolar moments (and not merely dipolar) [12,13]. This is because the standard picture of the screened dipolar local moments by conduction electrons may not be applicable to the multipolar cases. In fact, local moments in many heavy fermion systems carry multipolar moments because of strong spin-orbit coupling and crystal electric field effects [14-24]. The fate of such an unusual Kondo effect would also have ramifications in multipolar ordering induced by the same coupling, often dubbed "hidden order" due to the difficulty of detecting

Published by the American Physical Society under the terms of the Creative Commons Attribution 4.0 International license. Further distribution of this work must maintain attribution to the author(s) and the published article's title, journal citation, and DOI. it with conventional experimental probes [25,26]. An earlier investigation of this issue [27] suggests that when the quadrupolar moment of a single local impurity couples to conduction electrons, the resulting Kondo effect leads to a non-Fermi-liquid state. Here the orbital degrees of freedom of the conduction electrons are entangled with the fluctuations of quadrupolar moments while the spin quantum number of conduction electrons merely provides two separate channels for the scattering $[27,28]$. This model has been used for further exploration of non-Fermi-liquid physics.

In this paper, we investigate multipolar quantum impurity systems, where the local moment carries both quadrupolar and octupolar moments, and are coupled to conduction electrons. We are motivated by a series of experiments [14,29-31] performed on the cubic heavy fermion systems, $\operatorname{Pr}(\mathrm{TM})_{2} \mathrm{Al}_{20}$ $(\mathrm{TM}=\mathrm{Ti}, \mathrm{V})$, where the non-Kramers doublet of $\mathrm{Pr}^{3+}$ ions carry quadrupolar and octupolar moments. Here we consider a single multipolar impurity coupled to conduction electrons in generic symmetry-allowed orbital channels. Using perturbative renormalization group analysis, we establish the presence of more than one possible non-Fermi-liquid state, signified by the presence of nontrivial fixed points in the renormalization group flow. These include a novel non-Fermi-liquid state, which was not identified in previous studies, while the others are connected to the two-channel Kondo effect mentioned above. The newly discovered non-Fermi-liquid state is characterized by the absence of well-defined quasiparticles, and power-law behaviors in response functions that are more singular than the two-channel Kondo counterpart. In this case, quantum fluctuations of the multipolar moments are highly entangled with both the orbital and spin degrees of freedom of conduction electrons. This is in contrast to the two-channel Kondo model, where only the orbital is entangled with local moment fluctuations, while spin remains a spectator. Indeed, the discovered novel non-Fermi liquid is outside the paradigm 
of the multi-channel Kondo effect. We expect that our results would serve as a valuable starting point towards understanding non-Fermi-liquid states, quantum phase transitions, and critical phenomena associated with "hidden order" systems.

\section{MODELS}

We first consider the origin and nature of the local moments that arise in the archetypal multipolar heavy fermion system, as well as the source of the conduction electrons. We then present the subsequent "Kondo" Hamiltonians, highlighting the importance of the orbital dependence on the form of the Kondo coupling. In this aspect, we start by writing an effective (low-energy) Kondo Hamiltonian from pure symmetry considerations, without an emphasis on any particular microscopic model. This approach is attractive as it ensures that we are not restricted to any particular microscopic model to begin with, nor does it require us to precisely know the microscopic processes and mechanisms responsible for the low-energy interaction. As such, we are able to explore (symmetry-permitted) generic forms of Kondo couplings that can arise in this system. Nevertheless, to place our work in the context of previous quadrupolar Kondo models [27,28], we also present a microscopic inter-site Anderson model, which involves the hybridization of conduction electrons with the local moment $f$-electron states. Indeed, in the low-energy limit, this Anderson model reduces to our effective "Kondo" models, thus providing an explicit microscopic origin of our low-energy theory.

\section{A. Local moment physics of Pr ions}

An ideal representative example of multipolar heavy fermion systems is the cubic rare-earth family $\operatorname{Pr}(\mathrm{TM})_{2} \mathrm{Al}_{20}$ $(\mathrm{TM}=\mathrm{Ti}, \mathrm{V})$, where the localized multipolar degrees of freedom arise from $\operatorname{Pr} 4 f^{2}$ electrons. In these compounds, each $\mathrm{Pr}$ ion is surrounded by a Frank-Kasper (FK) cage of $\mathrm{Al}$ atoms (16 Al-atom polyhedra), which subjects the $f$ electrons to a crystalline electric field (CEF) of local $T_{d}$ symmetry. This CEF splits the $J=4$ multiplet of the $4 f^{2}$ electrons to yield ground states formed by a $\Gamma_{3 g}$ non-Kramers doublet. The $\Gamma_{3 g}$ states support time-reversal even quadrupolar moments $\mathcal{O}_{20}=$ $\frac{1}{2}\left(3 J_{z}^{2}-J^{2}\right), \mathcal{O}_{22}=\frac{\sqrt{3}}{2}\left(J_{x}^{2}-J_{y}^{2}\right)$, and time-reversal odd octupolar moment $\mathcal{T}_{x y z}=\frac{\sqrt{15}}{6} \overline{J_{x} J_{y} J_{z}}$, where the overline represents a fully symmetrized product. Using a pseudospin basis $\{|\uparrow\rangle,|\downarrow\rangle\}$ from the doublet (as described in Appendix A), we can write the multipolar moments in terms of an effective pseudospin-1/2 operator $\vec{S}=\left(S^{x}, S^{y}, S^{z}\right)$,

$$
S^{x}=-\frac{1}{4} \mathcal{O}_{22}, \quad S^{y}=-\frac{1}{4} \mathcal{O}_{20}, \quad S^{z}=\frac{1}{3 \sqrt{5}} \mathcal{T}_{x y z} .
$$

The XY components of the pseudospin vector represent the quadrupolar degrees of freedom, while the Ising direction describes the octupolar degree of freedom.

\section{B. Conduction electron model}

We now turn to the conduction electrons which, in this family, arise primarily from the $\mathrm{Al}$ atoms and the TM ions. Since our principal interest in this work is on the Kondo coupling of conduction electrons to localized Pr moments, we focus on the itinerant electrons emanating from the $\mathrm{Al}$ atoms, and ignore the contributions from the TM ions. This choice stems from the physically justifiable expectation that cage compounds well isolate the (Pr) atom located at the center of the cage from the rest of its surroundings. We now focus on a single $\mathrm{FK}$ cage of $\mathrm{Al}$ atoms. Due to the cubic symmetry of the FK cage, the eigenstates of the electrons hopping on a single cage can be organized into the irreducible representations of the local $T_{d}$ group. Importantly, each eigenmode $A_{1}, T_{2}, E, \ldots$ has the same symmetry character as $s, p, e_{g}, \ldots$ electron orbitals centered about the FK cage. We can thus interpret the eigenmodes as being equivalent to electron molecular orbitals that are located about the center of the cage, and the conduction Hamiltonian describes hopping between these molecular orbitals on different diamond sites. Equipped with these molecular orbitals, there is still, however, an ambiguity as to which orbitals contribute to the Fermi surface and thus to the form of the Kondo coupling. Recent de Haas-van Alphen (dHvA) studies [32] have suggested a localized Fermi pocket about the zone center for $\operatorname{PrTi}_{2} \mathrm{Al}_{20}$, although the orbital character of the Fermi surface is still unknown. We can nevertheless proceed by selecting conduction electrons in $e_{g}$-like and $p$-like molecular orbitals. This choice is plausible as the subsequently obtained tight-binding band structure recovers a well-localized Fermi surface about the zone center as described in Appendix B. More importantly, this selection of molecular orbitals is the simplest choice to obtain nontrivial Kondo couplings with the localized moments: $e_{g}$ orbitals yield the familiar two-channel Kondo model, while $p$ orbitals result in novel Kondo couplings that permit both conduction electron spin and orbital to participate in the quantum scattering events. For completeness, we note that the partially filled nature of the TM ions' $d$ orbitals do not contribute to the below described "Kondo" physics, as experimental resistivity [29] and dHvA [32] studies on the isostructural $\mathrm{La}(\mathrm{Ti}, \mathrm{V})_{2} \mathrm{Al}_{20}$ indicate that the responsibility lies with the $f$-electron moment arising from the Prions.

\section{C. $e_{g}$ orbital Kondo model}

We first consider conduction electrons residing in the $e_{g}$ orbitals. The form of the Kondo coupling to the multipolar moments is constrained by the local $T_{d}$ symmetry imposed by the FK cage; we detail in Appendix $\mathrm{C}$ the manner in which the multipolar moments and the conduction electron degrees of freedom transform under the generating elements of $T_{d}$. The symmetry-permitted coupling is of the form

$$
\begin{aligned}
& H_{Q}^{e_{g}}=J_{Q} c_{j, a, \alpha}^{\dagger}\left[S_{j}^{x} \sigma_{\alpha \beta}^{0} \otimes \tau_{a b}^{x}-S_{j}^{y} \sigma_{\alpha \beta}^{0} \otimes \tau_{a b}^{z}\right] c_{j, b, \beta}, \\
& H_{O}^{e_{g}}=-J_{O} S_{j}^{z} c_{j, a, \alpha}^{\dagger}\left[\sigma_{\alpha \beta}^{0} \otimes \tau_{a b}^{y}\right] c_{j, b, \beta},
\end{aligned}
$$

where repeated Latin (Greek) indices are implicit summations over orbital (spin) degrees of freedom, and $\tau$ is the usual spin-1/2 Pauli matrix describing the $e_{g}$ orbital degree of freedom $\left\{x^{2}-y^{2}, 2 z^{2}-x^{2}-y^{2}\right\}$ as listed in Appendix D. We note that $j$ is the site where the (impurity) multipolar moment resides. The $e_{g}$ orbitals introduce two Kondo couplings: quadrupolar $J_{Q}$ and octupolar $J_{O}$. We note that the coupling in Eq. (2) has precisely the same form as the two-channel Kondo 
model, where here the conduction electron spin plays the role of a channel index. Although the sign structure in Eq. (2) may seem dissimilar to the usual two-channel Kondo model, this is merely an artifact of the choice of multipolar basis; a unitary transformation of the multipolar moments' basis allows the familiar form to be recovered.

\section{D. p-orbital Kondo model}

We next turn to the problem of coupling $p$-orbital conduction electrons to the local moments. The symmetry allowed couplings are of the form

$$
\begin{aligned}
H_{Q 1}^{p}= & K_{Q 1} c_{j, a, \alpha}^{\dagger}\left[S_{j}^{x} \sigma_{\alpha \beta}^{0} \otimes \lambda_{a b}^{x^{2}-y^{2}}-S_{j}^{y} \sigma_{\alpha \beta}^{0} \otimes \lambda_{a b}^{2 z^{2}-x^{2}-y^{2}}\right] c_{j, b, \beta}, \\
H_{O}^{p}= & K_{O} S_{j}^{z} c_{j, a, \alpha}^{\dagger} \\
& \times\left[\sigma_{\alpha \beta}^{x} \otimes \lambda_{a b}^{y z, r}+\sigma_{\alpha \beta}^{y} \otimes \lambda_{a b}^{x z, r}+\sigma_{\alpha \beta}^{z} \otimes \lambda_{a b}^{x y, r}\right] c_{j, b, \beta}, \\
H_{Q 2}^{p}= & K_{Q 2} c_{j, a, \alpha}^{\dagger}\left[\left(\sqrt{3} S_{j}^{x}-S_{j}^{y}\right) \sigma_{\alpha \beta}^{x} \otimes \lambda_{a b}^{y z, i}\right. \\
& \left.+\left(\sqrt{3} S_{j}^{x}+S_{j}^{y}\right) \sigma_{\alpha \beta}^{y} \otimes \lambda_{a b}^{x z, i}+2 S_{j}^{y} \sigma_{\alpha \beta}^{z} \otimes \lambda_{a b}^{x y, i}\right] c_{j, b, \beta},
\end{aligned}
$$

where repeated Latin (Greek) indices are implicit summations over orbital (spin) degrees of freedom, and $\lambda$ are the SU(3) Gell-Mann matrices describing the orbital degree of freedom $\left\{p_{x}, p_{y}, p_{z}\right\}$ as listed in Appendix D. We note that $j$ is the site where the (impurity) multipolar moment resides. The interorbital hopping $\lambda^{a b}$ matrices include the superscript of $r$ and $i$, indicating whether it contains solely $r$ eal or imaginary matrix elements. This notation allows one to easily notice that the terms in Eq. (3) preserve time-reversal symmetry; the $\lambda^{a b}$ matrices containing $i$ superscript flip sign, and this sign change is compensated by the sign flip of the spin- $1 / 2$ Pauli matrices, $\vec{\sigma}$. The $p$ orbitals introduce three Kondo couplings: quadrupolar $K_{Q 1}, K_{Q 2}$ and octupolar $K_{O}$. Both of $H_{Q 1}^{p}$ and $H_{O}^{p}$ can be simply understood as the conduction electrons imitating the respective multipolar moments they are interacting with, in an effort to preserve the local $T_{d}$ symmetry. For instance, $H_{Q 1}^{p}$ describes the scattering phenomena of conduction electrons distorting their electron charge distribution to a quadrupolar form of $x^{2}-y^{2}$ and $2 z^{2}-x^{2}-y^{2}$ to interact with the timereversal even quadrupolar moments $J_{x}^{2}-J_{y}^{2}$ and $2 J_{z}^{2}-J_{x}^{2}-$ $J_{y}^{2}$, respectively. The spin degree of freedom remains as a spectator here. Similarly, $H_{O}^{p}$ describes the phenomena of conduction electrons forming an $x y z$-like octupolar moment from its spin and orbital degrees of freedom to interact with the time-reversal odd octupolar moment $J_{x} J_{y} J_{z}$. This simple picture is, however, difficult to apply to $H_{Q 2}^{p}$ as it involves the quadrupolar moments interacting with conduction electrons that undergo flip of both spin and orbital. One can minimally say that this term amplifies the quantum nature of the scattering event between the conduction electrons and local moment, as it involves both spin and orbital degrees of freedom.

\section{E. Combination of all orbitals: inclusion of $e_{g} \otimes p$ Kondo model}

Finally, we consider Kondo coupling involving the mixing of $e_{g}$ and $p$ orbitals. The actual form of the symmetryconstrained Hamiltonian is, however, not very illuminating, so it suffices here to state that we have three additional Kondo couplings: two involving interactions with the quadrupolar moments $\left(L_{Q 1}, L_{Q 2}\right)$ and one involving the octupolar moment $\left(L_{O}\right)$. This brings us to a grand total of eight coupling constants in the complete model: $J_{Q}, J_{O}$ from $e_{g}, K_{Q 1}, K_{Q 2}, K_{O}$ from $p$, and $L_{Q 1}, L_{Q 2}, L_{O}$ from $e_{g} \otimes p$. We present the complete Hamiltonian in Appendix I.

\section{F. Microscopic origin of Kondo couplings from an intersite Anderson model}

In considering an intersite Anderson model, it is necessary to consider the energy level scheme of the $f$ electrons. The $\mathrm{Pr}^{3+}$ ion can fluctuate from its $4 f^{2} \Gamma_{3}$ non-Kramers ground state to Kramers $4 f^{1}$ and $4 f^{3}$ excited states via hybridization with a bath of conduction electrons. For the purpose of this discussion, it suffices to consider the $f^{1}$ doublet excited levels, which is of $\Gamma_{7}$ symmetry (the $f^{3}$ state is assumed to be at much higher energy than the $f^{1}$ state, and therefore ignored $[27,28])$. The basis states of the $f^{1}$ level are presented in Appendix A. Group theoretically, a valence fluctuation from the $f^{2}\left(\Gamma_{3}\right)$ to $f^{1}\left(\Gamma_{7}\right)$ levels is only permitted by conduction electrons with specific symmetry: $\Gamma_{c}=\Gamma_{3} \otimes \Gamma_{7}=\Gamma_{8}$, where $\Gamma_{c}$ is the conduction electron irrep. In Refs. [27,28], the hybridizing conduction electrons were taken to be a $\Gamma_{8}$ quartet of partial waves, which were constructed from $l=3$ angular momentum states, which were spin-orbit coupled to produce $j=\frac{5}{2}, \frac{7}{2}$ manifolds. This analysis led to the storied two-channel Kondo model at low-energy. Instead, we can consider other means by which to construct the $\Gamma_{8}$ irreducible representation. One possibility is that of $\Gamma_{c}=\Gamma_{8}=e_{g} \otimes \frac{1}{2}$, where the $e_{g}$ orbitals transform as $\Gamma_{3}$ and $\frac{1}{2}$ is the spin- $1 / 2$ spinor states that transform as $\Gamma_{6}$. Such conduction orbitals, as will be shown, is the microscopic origin of the above $e_{g}$ Kondo model. Another possibility is that of $\Gamma_{c}=p \otimes \frac{1}{2}=$ $\Gamma_{8} \oplus \Gamma_{6}$, which can also mediate the $f^{2} \leftrightarrow f^{1}$ transition; here, $p$ transforms as a $\Gamma_{4}$ irrep. Thus group theoretically, conduction $e_{g}$ and $p$ orbitals equipped with spinor degrees of freedom are permitted to hybridize with the $\mathrm{Pr}^{3+}$ energy levels.

We now present an explicit demonstration of the above group theory discussion by considering a single four-site tetrahedron cage of $\mathrm{Al}$ atoms, each hosting atomic $p$ orbitals. The choice of atomic orbitals is justified from the valence electronic configuration of an $\mathrm{Al}$ atom $[\mathrm{Ne}] 3 s^{2} 3 p^{1}$. The tetrahedron geometry is indeed a simplification of the more complicated FK cage, but is valid as it still imposes the constraining $T_{d}$ symmetry of the cage. Considering a tight-binding hopping matrix on this single tetrahedron, the corresponding molecular orbitals (eigenstates) are decoupled as: $A_{1} \oplus E \oplus T_{1} \oplus 2 T_{2}$, where we use the $T_{d}$ irrep notation. Here $E$ and $T_{2}$ have $e_{g}$ and $p$ as its basis functions. In abiding with the $T_{d}$ symmetry, we can derive the following intersite Anderson model,

$$
H_{\mathrm{hyb}}=\sum_{\Delta, \omega, j, \mathcal{P}, \sigma} V_{\Delta \omega j \mathcal{P} \sigma}\left|f^{2}, \Delta\right\rangle\left\langle f^{1}, \omega\right| c_{j \mathcal{P} \sigma}+\text { H.c. }
$$

where $V_{\Delta \omega j \mathcal{P} \sigma}$ is the hybridization matrix element, $\Delta=\{1,2\}$ sums over the two non-Kramers $\Gamma_{3}$ ground states, $\omega=\{+,-\}$ runs over the two $f^{1}$ levels, $j=\{1,2,3,4\}$ goes over the 
four sites, $\mathcal{P}=\left\{p_{x}, p_{y}, p_{z}\right\}$ runs over the atomic $\mathrm{Al} p$ orbitals, and $\sigma=\{\uparrow, \downarrow\}$ denotes the conduction electron spin. Constrained by symmetry, the ninety-six hybridization elements are composed of four independent parameters; we present its relations in Appendix E. Rewriting the above Anderson model in terms of the molecular orbitals on the cage, and performing a Schrieffer-Wolff transformation, as described in Appendix F, we thus obtain (i) for the $e_{g}$ molecular orbitals, the isotropic two-channel Kondo model of Eq. (2) and (ii) the $p$ orbital Kondo model of Eq. (3). As we show next, starting with any of these terms would generate all the symmetry allowed interactions during the renormalization group (RG) procedure.

\section{RESULTS}

Equipped with the low-energy models of the previous section, we examine the RG fixed points of our models by performing perturbative $\mathrm{RG}$ analysis of the vertex function as described in Appendix G. We present the RG equations and delineate the fixed points/lines of the $e_{g}$ and $p$ orbital models, before presenting a schematic plot of the parameter space, and the location of the various fixed points/lines. Finally, we discuss the physical properties associated with each of these fixed points.

\section{A. Renormalization group analysis of $e_{g}$ model: two channel Kondo model}

The perturbative renormalization group analysis of Eq. (2) (i.e., the two-channel Kondo model) yields the following $\beta$ functions for the two coupling constants,

$$
\begin{aligned}
& \frac{d J_{Q}}{d \ln D}=2 J_{Q} J_{O}+2 J_{Q}\left(J_{Q}^{2}+J_{O}^{2}\right), \\
& \frac{d J_{O}}{d \ln D}=2 J_{Q}^{2}+4 J_{O} J_{Q}^{2},
\end{aligned}
$$

whose fixed points are the decoupled Gaussian fixed point $G^{*} \equiv\left(J_{Q}=J_{O}=0\right)$, and nontrivial fixed points $e_{g \pm}^{*} \equiv$ $\left(J_{Q}, J_{O}\right)=( \pm 1 / 2,-1 / 2) . e_{g \pm}^{*}$ are known as the Nozières fixed points, which were found in perturbative RG computations of the two-channel Kondo model [33]. We also obtain a line of fixed points characterized by $J_{Q}=0, J_{O} \neq 0$. The Gaussian fixed point is saddlelike (one relevant and one irrelevant eigendirections), while the fixed line has regions where the nearby flow is attractive $\left(J_{O}>0\right)$ and repulsive $\left(J_{O}<0\right)$. We present the RG flow diagram in Appendix H. The nontrivial fixed points are related to each other by the transformation of $S^{x, y} \rightarrow-S^{x, y}$; this transformation preserves the canonical commutation rules for the pseudospin- $1 / 2$ operators. The $\beta$ function and the characteristics of the fixed points/lines are precisely the same as the usual two-channel Kondo model. This leads to the same physical behavior and exponents, as will be discussed.

\section{B. Renormalization group analysis of $p$ orbital Kondo model}

The perturbative renormalization group analysis of Eq. (3) yields the following $\beta$ functions for the three coupling constants,

$$
\begin{aligned}
& \frac{d K_{Q 1}}{d \ln D}=6 K_{Q 2} K_{O}+K_{Q 1}\left(12 K_{Q 2}^{2}+6 K_{O}^{2}+2 K_{Q 1}^{2}\right) \\
& \frac{d K_{Q 2}}{d \ln D}=K_{O}\left(K_{Q 1}-\sqrt{3} K_{Q 2}\right)+K_{Q 2}\left(12 K_{Q 2}^{2}+6 K_{O}^{2}+2 K_{Q 1}^{2}\right), \\
& \frac{d K_{O}}{d \ln D}=4 K_{Q 1} K_{Q 2}-2 \sqrt{3} K_{Q 2}^{2}+K_{O}\left(24 K_{Q 2}^{2}+4 K_{Q 1}^{2}\right)
\end{aligned}
$$

whose fixed points are [in terms of $\left.\left(K_{Q 1}, K_{Q 2}, K_{O}\right)\right]$ the Gaussian fixed point $G^{*}=(0,0,0), p_{2 \mp}^{*}=\left(\mp \frac{1}{2 \sqrt{3}}, \pm \frac{1}{6}, \frac{1}{2 \sqrt{3}}\right)$, and $p_{1 \mp}^{*}=\left(\mp \frac{1}{2 \sqrt{6}}, \mp \frac{1}{12 \sqrt{2}},-\frac{1}{4 \sqrt{3}}\right)$. The nontrivial fixed points are stable and attractive. We present a representative RG flow diagram in Appendix $\mathrm{H}$, where we depict the flow about the $p_{1 \mp}^{*}$ and $p_{2 \mp}^{*}$ fixed points. We also obtain a fixed line characterized by $K_{O} \neq 0\left(K_{Q 1}=K_{Q 2}=0\right)$, which is analogous to the fixed line from the usual spin-Kondo model. Just as in that instance, it describes an Ising-like interaction between the conduction electron and local moment. The subtlety here is that the Ising degree of freedom is octupolar, as opposed to dipolar in the usual Kondo problem.

\section{Renormalization group analysis of $e_{g} \otimes p$ orbital Kondo model}

For brevity, we present the complete corresponding eight RG flow equations in Appendix I, and also include the complete $\beta$ functions for the $e_{g}$ and $p$-only couplings, which have been modified by the inclusion of these three additional couplings. The solutions to the eight $\beta$ functions provide three extended lines of stable fixed points, two unstable fixed points, and an Ising plane of fixed points. The Ising plane is a generalization of the Ising "line" of fixed points of the $e_{g}$ and $p$ only models; specifically, all the quadrupolar coupling constants are zero, while the octupolar couplings are nonvanishing. We focus on the three stable (from the perspective of linear stability analysis) lines of interest, each of which are parametrized by a single coupling parameter. We present in Fig. 1 an illustrative schematic of the stable lines of fixed points to help conceptualize their relation to each other in the eight-dimensional parameter space. Importantly, the three connective lines of fixed points depicted by Lines $\mathcal{N}$ (red), $\overline{\mathcal{N}}$ (blue), and $\mathcal{P}$ (green) are described by a single coupling parameter and include (depending on the line of interest) some of the $e_{g}$-only and $p$-only fixed points.

(i) Line $\mathcal{N}$-denoted as a red curve in Fig. 1 -is parametrized by single coupling parameter, $J_{Q} \in[0,1 / 2]$ (density of $e_{g}$ orbitals coupling to the quadrupolar moments), and includes the Nozières $e_{g+}^{*}$ fixed point, and a $p$-orbital-only fixed point $p_{2+}^{*}$.

(ii) Line $\overline{\mathcal{N}}$-denoted as a blue curve in Fig. 1-is parametrized by single coupling parameter, $J_{Q} \in[-1 / 2,0]$ (density of $e_{g}$ orbitals coupling to the quadrupolar moments), and includes the related-to-line $\mathcal{N} e_{g-}^{*}$ and $p_{2-}^{*}$-orbital-only fixed points. Line $\overline{\mathcal{N}}$ is mapped to Line $\mathcal{N}$ by taking $J_{Q} \rightarrow-J_{Q}$.

(iii) Line $\mathcal{P}$-denoted as a green curve in Fig. 1is parametrized by single coupling parameter, $K_{Q 1} \in$ $\left[-\frac{1}{2 \sqrt{6}}, \frac{1}{2 \sqrt{6}}\right]$ (density of $p$ orbitals coupling to the quadrupolar 


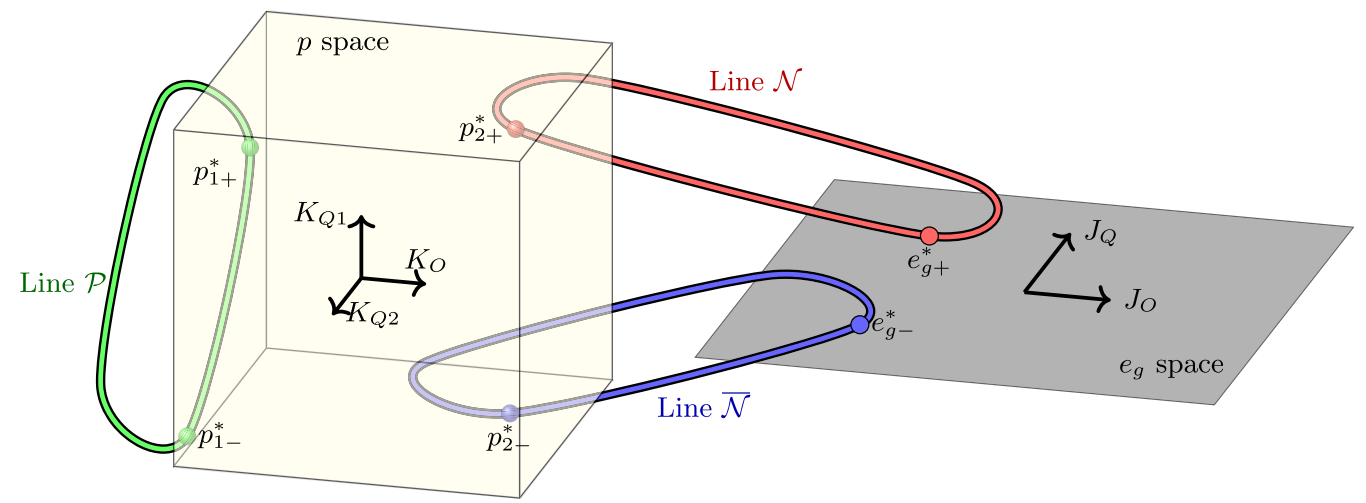

FIG. 1. Schematic plot denoting RG fixed lines and points in eight dimensional parameter space of the complete multipolar quantum impurity model. The yellow-shaded cube denotes the $p$-model space, with its corresponding fixed points at $p_{1 \mp}^{*}$ and $p_{2 \mp}^{*}$ indicated by green spheres, and red and blue spheres, respectively. The $e_{g}$ space is depicted by the grey-shaded plane, which includes the Nozières fixed points $e_{g \pm}^{*}$. The connective Lines $\mathcal{N}, \overline{\mathcal{N}}, \mathcal{P}$ are denoted by red, blue and green one-dimensional curves, respectively, which reside in the three extra dimensions (that are not shown in the figure).

moments), and includes two (related to each other) distinct $p$-orbital-only fixed points $p_{1 \mp}^{*}$. These $p$-orbital-only fixed points are distinct from the ones located in Lines $\mathcal{N}$ and $\overline{\mathcal{N}}$.

\section{Physical properties: specific heat and resistivity}

The nature of the fixed points (lines) can be better understood by examining their influence on the physical properties of the conduction electrons, namely specific heat $c_{v}$ and electrical resistivity $\rho$. Since each of the isolated fixed points of the $e_{g}$-only and $p$-only models are included in the connective lines, it suffices to discuss the physical properties of each of the stable lines. We follow the perturbative approach used in Ref. [34] to compute these two observables, and describe in Appendix $\mathrm{J}, \mathrm{K}$, and $\mathrm{L}$ the schemes used in obtaining the exponents. We present a summary of the scaling behavior of the specific heat and resistivity in the three connective lines in Table I. We note that the total scattering/decay rate $\left(1 / \tau_{\text {tot }}\right)$ is directly proportional to the resistivity, which allows us to infer the sustainability of the quasiparticle picture in these models.

Lines $\mathcal{N}$ and $\overline{\mathcal{N}}$ provide the same scaling behavior as the usual two-channel Kondo model at two-loop calculation. This is not altogether surprising, as these lines include the $e_{g}$ fixed points. One may imagine these lines as being an extension of the Nozières like fixed points in the eight-dimensional parameter space. Based on our perturbative analysis, we can thus claim that the low-energy physics described by lines $\mathcal{N}$ and $\overline{\mathcal{N}}$ is precisely the same as the two-channel Kondo model, i.e., we obtain the non-Fermi liquid of the two-channel model.

Line $\mathcal{P}$ (and the $p$-only fixed points it includes) is a novel line of fixed points, with distinct non-Fermi-liquid behavior.

TABLE I. Specific heat and resistivity scaling behaviors (at third order in Kondo coupling) associated with lines of fixed points $\mathcal{N}, \overline{\mathcal{N}}$, and $\mathcal{P}$. $\alpha_{1,2,3,4}$ denote proportionality constants.

\begin{tabular}{ccc}
\hline \hline Observable & Lines $\mathcal{N}$ and $\overline{\mathcal{N}}$ & Line $\mathcal{P}$ \\
\hline$c_{v}$ & $\sim T+\alpha_{1} T^{2}$ & $\sim T^{\frac{1}{4}}+\alpha_{2} T^{\frac{1}{2}}$ \\
$\rho$ & $\sim$ const. $+\alpha_{3} T$ & $\sim$ const. $+\alpha_{4} T^{\frac{1}{4}}$ \\
\hline \hline
\end{tabular}

Specifically, as seen in Table I, it has more singular behavior in its scaling exponents for both the specific heat and resistivity, as compared to the usual two-channel Kondo model. For this line, the scattering rate is much larger $\left(\sim T^{1 / 4}\right)$ than the quasiparticle energy $(\sim T)$; this is equivalent to stating that the time it takes for the quasiparticle (eigenstate) to decay is much shorter than the quasiparticle (eigenstate's) lifetime. As such, the breakdown of the Landau picture is dramatic along this line, leading to the absence of well-defined quasiparticles and the emergence of an exotic metallic phase with nontrivial lowlying excitations. We note that the scaling behaviors discussed here are valid up to third order in perturbative renormalization group analysis and as a consequence, they do not match up with the exponents from more sophisticated (and exact) approaches; for instance, in the known two-channel Kondo model, Bethe ansatz exact solution yields a logarithmically singular scaling to the specific heat $c_{v} \sim T \ln T[5,35]$, and boundary CFT [36] predicts the resistivity is of the form $\rho \sim$ const. $+\sqrt{T}$. Higher-order contributions may thus change the exponents reported above, however, we expect them to remain singular (as is the case for the two-channel Kondo model [37]).

\section{DISCUSSIONS}

In this work, we identified novel non-Fermi-liquid states in multipolar quantum impurity systems, where conduction electrons interact with local quadrupolar and octupolar moments. From our perturbative RG analysis, we found that low temperature behaviors of such systems can be governed by more than one possible non-Fermi-liquid states depending on the bare parameters of the systems. Such novel behaviors stem from the presence of two kinds of stable fixed points in the RG flow, one corresponding to the two channel Kondo problem and the other a newly discovered non-Fermi-liquid state with highly singular physical properties. Hence, drawing inspiration from heavy fermion materials where nonthermal parameters can be used to tune the ground state [38], if the bare parameters of the systems are changed by pressure or any other physical means, one would see a quantum phase transition between the two distinct non-Fermi-liquid phases at zero temperature, 
and a crossover at low temperatures. For instance, applying a hydrostatic pressure [18] would be means to compress the FK cage and thus increase the hybridization amplitudes (while preserving the $T_{d}$ symmetry of the FK cage). Another means would be via chemical substitution of the transition metal in (for example) $\mathrm{PrTi}_{2} \mathrm{Al}_{20}$ with Vanadium; this plays the role of a chemical pressure to compress the unit cell [29]. Both such physical and chemical pressures can thus be used to increase the hybridization amplitudes (and subsequently the Kondo couplings), and allow exploration of the coupling parameter space and the non-Fermi-liquid ground states.

While we solved a single multipolar quantum impurity system, our results imply possibly striking consequences for the Kondo lattice model and heavy fermion systems involving "hidden order". In particular, the conventional heavy fermion phase diagram must be reimagined as the strong-coupling limit may not merely be Fermi-liquid-like, but non-Fermiliquid-like with singular scaling in its response functions. Moreover, this work stresses the importance of incorporating conduction electron orbitals with different symmetries in determining the ultimate low energy ground states. This is highly suggestive that the inclusion of conduction electron orbitals with different symmetries in multipolar quantum impurity systems may be a new avenue to engineer emergent nonFermi-liquid states.

There are fascinating aspects of this work that still require further investigation. Firstly, the perturbative renormalization group approach evoked here establishes the existence of new non-Fermi-liquid states, signified by the nontrivial fixed points in the RG flow. The next natural step would be to confront this problem, in the manner of the conventional Kondo problem, with boundary CFT [37,39-41] or Bethe ansatz approaches [35,42-44]. It would be interesting to find the exact exponents of the above physical properties' scaling, so as to rigorously identify the nature of the novel non-Fermi liquid. Secondly, extension of this single impurity study to a generalized lattice of localized moments would be an interesting direction to pursue [45]. Finally, it would be intriguing to study the single multipolar impurity with scanning electron microscopy (STM) techniques, which have been historically fruitful endeavors in detecting the Kondo resonance in singlechannel Kondo systems [46,47]. Extension of previous theoretical predictions in multichannel Kondo systems (which suggest a characteristic multichannel Kondo resonance [48]) to the multipolar impurity model considered in this work, as well as experimental investigations, may help to provide better understanding of the nature of the unusual non-Fermi-liquid ground states.

\section{ACKNOWLEDGMENTS}

This work was supported by NSERC of Canada. Y.B.K. is supported by the Killam Research Fellowship of the Canada Council for the Arts. We thank SungBin Lee for insightful discussions on the molecular orbital bases of the conduction electrons. We are grateful to Wonjune Choi for illuminating discussions on the Kondo problem. We thank Mingxuan $\mathrm{Fu}$ for many helpful discussions on the manuscript.

\section{APPENDIX A: BASIS STATES OF MULTIPOLAR $f$ ELECTRONS}

The ground state of the $T_{d}$ subjected $f$ electrons is a nonKramers doublet which, written in the $\left|J_{z}\right\rangle$ basis, is

$$
\begin{aligned}
& \Gamma_{3}^{(1)}=\frac{1}{2} \sqrt{\frac{7}{6}}|4\rangle-\frac{1}{2} \sqrt{\frac{5}{3}}|0\rangle+\frac{1}{2} \sqrt{\frac{7}{6}}|-4\rangle, \\
& \Gamma_{3}^{(2)}=\frac{1}{\sqrt{2}}|2\rangle+\frac{1}{\sqrt{2}}|-2\rangle .
\end{aligned}
$$

We can construct a pseudospin-1/2 basis from these $\Gamma_{3 g}$ $(\{|\uparrow\rangle,|\downarrow\rangle\})$ as

$$
|\uparrow\rangle=\frac{1}{\sqrt{2}}\left[\left|\Gamma_{3}^{(1)}\right\rangle+i\left|\Gamma_{3}^{(2)}\right\rangle\right], \quad|\downarrow\rangle=\frac{1}{\sqrt{2}}\left[i\left|\Gamma_{3}^{(1)}\right\rangle+\left|\Gamma_{3}^{(2)}\right\rangle\right],
$$

which allows the multipolar moments to be neatly written as an effective pseudospin-1/2 operator $\vec{S}=\left(S^{x}, S^{y}, S^{z}\right)$ (see main text).

The $4 f^{1}$ excited state belongs to the $\Gamma_{7}$ irrep. with basis states,

$$
\left|\Gamma_{7}, \pm\right\rangle=\sqrt{\frac{1}{6}}\left| \pm \frac{5}{2}\right\rangle-\sqrt{\frac{5}{6}}\left|\mp \frac{3}{2}\right\rangle .
$$

\section{APPENDIX B: SLATER-KOSTER TIGHT-BINDING MODEL}

We model the conduction electrons kinetic term using the Slater-Koster method of tight binding for the $p$ and $e_{g}$ orbitals located on sublattice A and B of the diamond lattice. To simplify the matrix structure of the kinetic term we retain only first nearest neighbor hoppings, and allow only like-to-like orbital hopping. These assumptions still ensure a localized Fermi surface about the zone center, and yield kinetic eigenvectors (and eigenvalues) that do not mix different orbitals together, as seen below. This ensures that the form of "Kondo" coupling presented in the next section is unaffected by rewriting it in terms of the kinetic eigenvectors.

We present in Fig. 2 a plot of the tight-binding band structure, as well as a plot of a Fermi surface localized about the band center (the Fermi energy is set at the dotted line in the band structure) showing the localized Fermi surface about the zone center. Moreover, focusing on the bands closest to the Fermi surface, one can see a set of nearly degenerate bands, which have approximately the same bandwidth. We note that it is reasonable to take the bands to be degenerate in momentum space, as any nondegeneracy manifests itself in terms of an irrelevant perturbation (in the renormalization group sense) for the two-channel Kondo model [49].

The corresponding eigenvectors and eigenvalues of the Slater-Koster tight-binding model involving $p$ and $e_{g}$ orbitals, and assuming a Fermi surface localized about the zone center, i.e., $|\vec{k}| \ll 1$,

$$
\begin{aligned}
& c_{ \pm 1}=\frac{1}{\sqrt{2}}\left(c_{x, A} \pm c_{x, B}\right), \quad c_{ \pm 2}=\frac{1}{\sqrt{2}}\left(c_{y, A} \pm c_{y, B}\right), \\
& c_{ \pm 3}=\frac{1}{\sqrt{2}}\left(c_{z, A} \pm c_{z, B}\right),
\end{aligned}
$$




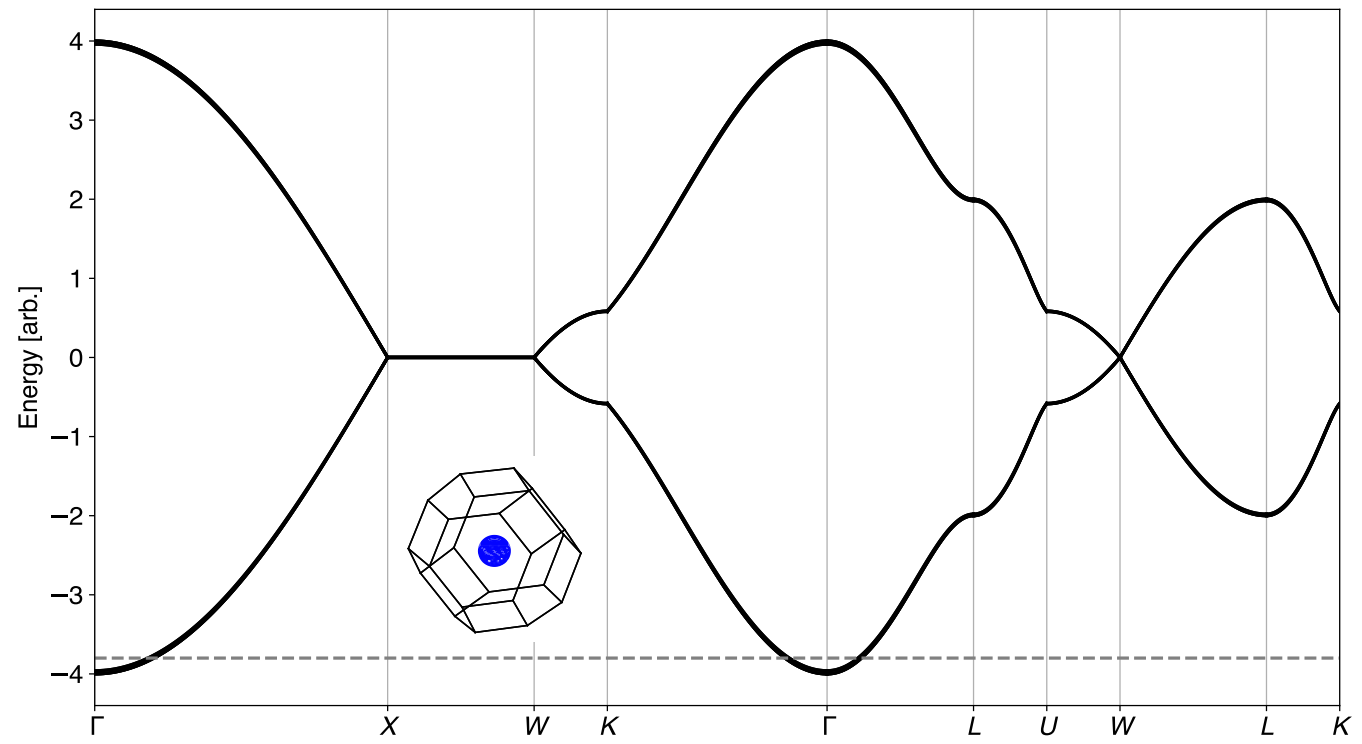

FIG. 2. Slater-Koster tight-binding band structure of $e_{g} \otimes T_{2}$ model, and Fermi level indicated by a dotted line in a band structure for $t=1.5, t^{\prime}=0$, and $s_{T_{2}}=p_{T_{2}}=1, p_{e}=1, d_{e}=0.2$. (Inset) Fermi surface (blue) depicted in first Brillouin zone (black).

$$
\begin{aligned}
& c_{ \pm 4}= \frac{1}{\sqrt{2}}\left(c_{x^{2}-y^{2}, A} \pm c_{x^{2}-y^{2}, B}\right) \\
& c_{ \pm 5}= \frac{1}{\sqrt{2}}\left(c_{2 z^{2}-x^{2}-y^{2}, A} \pm c_{2 z^{2}-x^{2}-y^{2}, B}\right) \\
& E_{ \pm 1, \pm 2, \pm 3}= \pm s_{T_{2}}\left(4-\frac{k^{2}}{4}\right) \\
& E_{ \pm 4, \pm 5}= \pm \frac{d_{e}+2 p_{e}}{3}\left(4-\frac{k^{2}}{4}\right),
\end{aligned}
$$

where $s_{T_{2}}$ is the $\sigma$ overlap for the $T_{2}$ orbitals; and $p_{e}$ and $d_{e}$ are the $\pi$ and $\delta$ overlaps for the $e_{g}$ orbitals. As seen in Eq. (B1), the different orbitals do not mix with each other in the tight-binding eigenbasis. We stress that the details of the tight-binding model presented here are not employed in this work; the eigenenergies in Fig. 2 merely serve to demonstrate that a localized Fermi pocket is possible with these choice of molecular orbitals. Throughout our calculations, we only utilize the fact that there is a finite density of states at the Fermi level.

\section{APPENDIX C: SYMMETRY PROPERTIES OF MULTIPOLAR MOMENTS AND CONDUCTION ELECTRONS}

We consider here the symmetry properties of the multipolar moments, and then discuss conduction electrons. We note the spin of the conduction electrons is denoted by $\vec{\sigma}$. We recall the two generating elements of the $T_{d}$ group, namely, $S_{4 z}$ and $C_{31}$. In real $\mathbb{R}^{3}$ space, the matrix representations of $S_{4 z}$ and $C_{31}$ are

$$
S_{4 z}=\left[\begin{array}{ccc}
0 & -1 & 0 \\
1 & 0 & 0 \\
0 & 0 & 1
\end{array}\right] \cdot \mathbb{I}, \quad C_{31}=\left[\begin{array}{lll}
0 & 0 & 1 \\
1 & 0 & 0 \\
0 & 1 & 0
\end{array}\right],
$$

where $\mathbb{I}$ denotes spatial inversion $(x, y, z) \rightarrow(-x,-y,-z)$. We also consider time-reversal $(\Theta)$ symmetry. We present in Table II how the multipolar moments, and conduction electrons' spin and orbitals transform under the $T_{d}$ point group and time reversal $(\Theta)$.

\section{APPENDIX D: PSEUDOSPIN REPRESENTATION OF CONDUCTION ORBITALS}

Here we present the matrices that are used in describing the orbital degrees of freedom in the main text: (i) $\tau$ is the usual Pauli- $2 \times 2$ matrix spanning the vector space $e_{g}$ orbitals. (ii) $\lambda$ are the usual Gell-Mann $3 \times 3$ matrices spanning the vector space $p$ orbitals. (iii) $\kappa$ are generalized Gell-Mann $5 \times$ 5 matrices spanning the vector space of $e_{g} \otimes p$ orbitals (we only list a subset of the $5 \times 5$ matrices used in this work). The

TABLE II. Transformation of multipolar order parameters, and conduction electron degrees of freedom under generating elements of $T_{d}$ $\left(\mathcal{S}_{4 z}, \mathcal{C}_{31}\right)$, and time reversal $(\Theta)$. We use the notation of $e_{g 1}=\left(2 z^{2}-x^{2}-y^{2}\right) / \sqrt{3}$ and $e_{g 2}=x^{2}-y^{2}$ for the $d$ orbitals.

\begin{tabular}{lccc}
\hline \hline Operator & $\Theta$ & $\mathcal{S}_{4 z}$ & $\mathcal{C}_{31}$ \\
\hline$\left(S^{x}, S^{y}, S^{z}\right)$ & $\left(S^{x}, S^{y},-S^{z}\right)$ & $\left(-S^{x}, S^{y},-S^{z}\right)$ & $\left(-\frac{1}{2} S^{x}+\frac{\sqrt{3}}{2} S^{y},-\frac{\sqrt{3}}{2} S^{x}-\frac{1}{2} S^{y}, S^{z}\right)$ \\
$\left(\sigma^{x}, \sigma^{y}, \sigma^{z}\right)$ & $\left(-\sigma^{x},-\sigma^{y},-\sigma^{z}\right)$ & $\left(-\sigma^{y}, \sigma^{x}, \sigma^{z}\right)$ & $\left(\sigma^{z}, \sigma^{x}, \sigma^{y}\right)$ \\
$\left(p_{x}, p_{y}, p_{z}\right)$ & $\left(p_{x}, p_{y}, p_{z}\right)$ & $\left(p_{y},-p_{x},-p_{z}\right)$ & $\left(p_{z}, p_{x}, p_{y}\right)$ \\
$\left(e_{g 1}, e_{g 2}\right)$ & $\left(e_{g 1}, e_{g 2}\right)$ & $\left(e_{g 1},-e_{g 2}\right)$ & $\left(-\frac{\sqrt{3}}{2} e_{g 2}-\frac{1}{2} e_{g 1},-\frac{1}{2} e_{g 2}+\frac{\sqrt{3}}{2} e_{g 1}\right)$ \\
\hline \hline
\end{tabular}


matrices are represented in the following basis where $e_{g 1}=\left(2 z^{2}-x^{2}-y^{2}\right) / \sqrt{3}$ and $e_{g 2}=x^{2}-y^{2}$. The “..." symbolically represents a complex number.

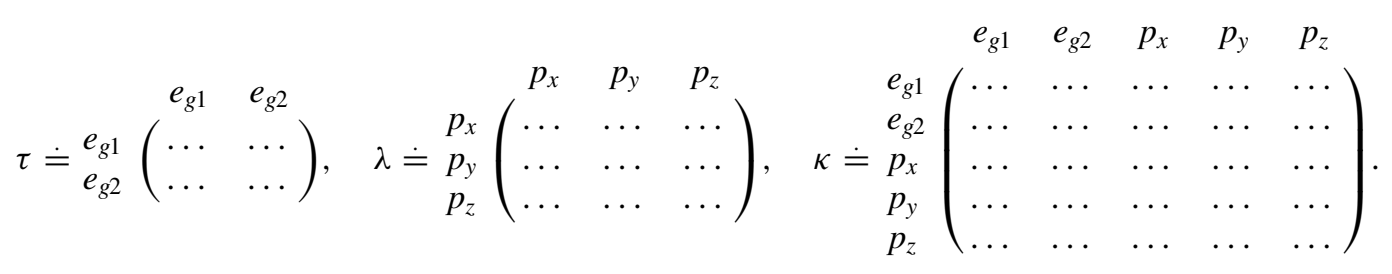

In this basis, we have

$$
\begin{aligned}
\tau^{x} \doteq\left(\begin{array}{cc}
0 & 1 \\
1 & 0
\end{array}\right) ; \quad \tau^{y} \doteq\left(\begin{array}{cc}
0 & -i \\
i & 0
\end{array}\right) ; \quad \tau^{z} \doteq\left(\begin{array}{cc}
1 & 0 \\
0 & -1
\end{array}\right) \\
\lambda^{0} \doteq\left(\begin{array}{ccc}
1 & 0 & 0 \\
0 & 1 & 0 \\
0 & 0 & 1
\end{array}\right) ; \quad \lambda^{x^{2}-y^{2}} \doteq\left(\begin{array}{ccc}
1 & 0 & 0 \\
0 & -1 & 0 \\
0 & 0 & 0
\end{array}\right) ; \quad \lambda^{2 z^{2}-x^{2}-y^{2}} \doteq \frac{-1}{\sqrt{3}}\left(\begin{array}{ccc}
-1 & 0 & 0 \\
0 & -1 & 0 \\
0 & 0 & 2
\end{array}\right) ; \\
\lambda^{x y, r} \doteq\left(\begin{array}{ccc}
0 & 1 & 0 \\
1 & 0 & 0 \\
0 & 0 & 0
\end{array}\right) ; \quad \lambda^{y z, r} \doteq\left(\begin{array}{ccc}
0 & 0 & 0 \\
0 & 0 & 1 \\
0 & 1 & 0
\end{array}\right) ; \quad \lambda^{x z, r} \doteq\left(\begin{array}{ccc}
0 & 0 & 1 \\
0 & 0 & 0 \\
1 & 0 & 0
\end{array}\right) ; \\
\lambda^{x y, i} \doteq\left(\begin{array}{ccc}
0 & -i & 0 \\
i & 0 & 0 \\
0 & 0 & 0
\end{array}\right) ; \quad \lambda^{y z, i} \doteq\left(\begin{array}{ccc}
0 & 0 & 0 \\
0 & 0 & -i \\
0 & i & 0
\end{array}\right) ; \quad \lambda^{x z, i} \doteq\left(\begin{array}{ccc}
0 & 0 & -i \\
0 & 0 & 0 \\
i & 0 & 0
\end{array}\right)
\end{aligned}
$$$$
\kappa^{1 x, r} \doteq\left(\begin{array}{ccccc}
0 & 0 & 1 & 0 & 0 \\
0 & 0 & 0 & 0 & 0 \\
1 & 0 & 0 & 0 & 0 \\
0 & 0 & 0 & 0 & 0 \\
0 & 0 & 0 & 0 & 0
\end{array}\right) ; \quad \kappa^{1 y, r} \doteq\left(\begin{array}{ccccc}
0 & 0 & 0 & 1 & 0 \\
0 & 0 & 0 & 0 & 0 \\
0 & 0 & 0 & 0 & 0 \\
1 & 0 & 0 & 0 & 0 \\
0 & 0 & 0 & 0 & 0
\end{array}\right)
$$$$
\kappa^{1 z, r} \doteq\left(\begin{array}{ccccc}
0 & 0 & 0 & 0 & 1 \\
0 & 0 & 0 & 0 & 0 \\
0 & 0 & 0 & 0 & 0 \\
0 & 0 & 0 & 0 & 0 \\
1 & 0 & 0 & 0 & 0
\end{array}\right) \quad \kappa^{2 x, r} \doteq\left(\begin{array}{ccccc}
0 & 0 & 0 & 0 & 0 \\
0 & 0 & 1 & 0 & 0 \\
0 & 1 & 0 & 0 & 0 \\
0 & 0 & 0 & 0 & 0 \\
0 & 0 & 0 & 0 & 0
\end{array}\right)
$$$$
\kappa^{2 y, r} \doteq\left(\begin{array}{ccccc}
0 & 0 & 0 & 0 & 0 \\
0 & 0 & 0 & 1 & 0 \\
0 & 0 & 0 & 0 & 0 \\
0 & 1 & 0 & 0 & 0 \\
0 & 0 & 0 & 0 & 0
\end{array}\right) ; \quad \kappa^{2 z, r} \doteq\left(\begin{array}{ccccc}
0 & 0 & 0 & 0 & 0 \\
0 & 0 & 0 & 0 & 1 \\
0 & 0 & 0 & 0 & 0 \\
0 & 0 & 0 & 0 & 0 \\
0 & 1 & 0 & 0 & 0
\end{array}\right)
$$$$
\kappa^{1 x, i} \doteq\left(\begin{array}{ccccc}
0 & 0 & -i & 0 & 0 \\
0 & 0 & 0 & 0 & 0 \\
i & 0 & 0 & 0 & 0 \\
0 & 0 & 0 & 0 & 0 \\
0 & 0 & 0 & 0 & 0
\end{array}\right) ; \quad \kappa^{1 y, i}=\left(\begin{array}{ccccc}
0 & 0 & 0 & -i & 0 \\
0 & 0 & 0 & 0 & 0 \\
0 & 0 & 0 & 0 & 0 \\
i & 0 & 0 & 0 & 0 \\
0 & 0 & 0 & 0 & 0
\end{array}\right)
$$$$
\kappa^{1 z, i} \doteq\left(\begin{array}{ccccc}
0 & 0 & 0 & 0 & -i \\
0 & 0 & 0 & 0 & 0 \\
0 & 0 & 0 & 0 & 0 \\
0 & 0 & 0 & 0 & 0 \\
i & 0 & 0 & 0 & 0
\end{array}\right) ; \quad \kappa^{2 x, i} \doteq\left(\begin{array}{ccccc}
0 & 0 & 0 & 0 & 0 \\
0 & 0 & -i & 0 & 0 \\
0 & i & 0 & 0 & 0 \\
0 & 0 & 0 & 0 & 0 \\
0 & 0 & 0 & 0 & 0
\end{array}\right)
$$$$
\kappa^{2 y, i} \doteq\left(\begin{array}{ccccc}
0 & 0 & 0 & 0 & 0 \\
0 & 0 & 0 & -i & 0 \\
0 & 0 & 0 & 0 & 0 \\
0 & i & 0 & 0 & 0 \\
0 & 0 & 0 & 0 & 0
\end{array}\right) ; \quad \kappa^{2 z, i} \doteq\left(\begin{array}{ccccc}
0 & 0 & 0 & 0 & 0 \\
0 & 0 & 0 & 0 & -i \\
0 & 0 & 0 & 0 & 0 \\
0 & 0 & 0 & 0 & 0 \\
0 & i & 0 & 0 & 0
\end{array}\right) \text {. }
$$ 


\section{APPENDIX E: MICROSCOPIC INTERSITE ANDERSON MODEL}

As discussed in the main text, we consider a four Al-atom tetrahedron cage which has the same $T_{d}$ point group. For the sake of clarity, we consider a Pr atom centered at the origin $(0,0,0)$ and with the four $\mathrm{Al}$ atoms on the corners of the cube (to form the tetrahedron): $(1):(1,1,1),(2):(1,-1,-1)$, (3) : $(-1,1,-1)$, and $(4):(-1,-1,1)$. Under the applied transformation using the symmetry generators of the $T_{d}$ group, the four sites get mapped to each other. Under $\mathcal{C}_{31}:$ (1) $\rightarrow$ $(1),(2) \rightarrow(4),(3) \rightarrow(2)$, and (4) $\rightarrow$ (3). Under $\mathcal{S}_{4 z}:(1) \rightarrow$
$(3)$, (2) $\rightarrow(1)$, (3) $\rightarrow$ (4), and (2) $\rightarrow$ (3). The following Table III lists the ninety-six hybridization matrix elements. As seen, they are composed of four independent parameters: $\mathcal{V}_{1,2,3,4}$

\section{APPENDIX F: SCHRIEFFER-WOLFF (SW) TRANSFORMATION}

The SW transformation is a means to perturbatively diagonalize a general interacting Hamiltonian using a unitary transformation. The transformation also results in renormal-

TABLE III. Intersite hybridization matrix elements $V_{\Delta \omega j \mathcal{P} \sigma}$.

\begin{tabular}{|c|c|c|c|c|c|}
\hline$f^{2}$ State: $\Delta$ & $f^{1}$ State: $\omega$ & Site $j$ & Atomic orbital: $\mathcal{P}$ & $c$ spin: $\sigma$ & $V_{\Delta \omega j \mathcal{P} \sigma}$ \\
\hline (1) & + & 1 & $x$ & $\uparrow$ & $\frac{1}{4}(i \sqrt{3}+1)\left(\mathcal{V}_{2}-i \mathcal{V}_{1}+i \mathcal{V}_{4}+\mathcal{V}_{3}\right)$ \\
\hline (1) & + & 1 & $x$ & $\downarrow$ & $\frac{1}{4}(-i+\sqrt{3})\left(-i \mathcal{V}_{2}+\mathcal{V}_{1}+\mathcal{V}_{4}+i \mathcal{V}_{3}\right)$ \\
\hline (1) & + & 1 & $y$ & $\uparrow$ & $\frac{1}{4}(i+\sqrt{3})\left(\mathcal{V}_{2}+\mathcal{V}_{1}+\mathcal{V}_{4}+\mathcal{V}_{3}\right)$ \\
\hline (1) & + & 1 & $y$ & $\downarrow$ & $\frac{1}{4} i(i+\sqrt{3})\left(\mathcal{V}_{2}-\mathcal{V}_{1}+\mathcal{V}_{4}-\mathcal{V}_{3}\right)$ \\
\hline (1) & + & 1 & $z$ & $\uparrow$ & $\mathcal{V}_{1}$ \\
\hline (1) & + & 1 & $z$ & $\downarrow$ & $\mathcal{V}_{2}$ \\
\hline (1) & + & 2 & $x$ & $\uparrow$ & $\frac{1}{4}(i \sqrt{3}+1)\left(\mathcal{V}_{2}+i \mathcal{V}_{1}-i \mathcal{V}_{4}+\mathcal{V}_{3}\right)$ \\
\hline (1) & + & 2 & $x$ & $\downarrow$ & $-\frac{1}{4} i(-i+\sqrt{3})\left(\mathcal{V}_{2}-i\left(\mathcal{V}_{1}+\mathcal{V}_{4}-i \mathcal{V}_{3}\right)\right)$ \\
\hline (1) & + & 2 & $y$ & $\uparrow$ & $\frac{1}{4}(i+\sqrt{3})\left(\mathcal{V}_{2}-\mathcal{V}_{1}-\mathcal{V}_{4}+\mathcal{V}_{3}\right)$ \\
\hline (1) & + & 2 & $y$ & $\downarrow$ & $\frac{1}{4}(1-i \sqrt{3})\left(\mathcal{V}_{2}+\mathcal{V}_{1}-\mathcal{V}_{4}-\mathcal{V}_{3}\right)$ \\
\hline (1) & + & 2 & $z$ & $\uparrow$ & $\mathcal{V}_{4}$ \\
\hline (1) & + & 2 & $z$ & $\downarrow$ & $\mathcal{V}_{3}$ \\
\hline (1) & + & 3 & $x$ & $\uparrow$ & $\frac{1}{4}(i \sqrt{3}+1)\left(\mathcal{V}_{2}+i \mathcal{V}_{1}-i \mathcal{V}_{4}+\mathcal{V}_{3}\right)$ \\
\hline (1) & + & 3 & $x$ & $\downarrow$ & $\frac{1}{4}(-i+\sqrt{3})\left(i \mathcal{V}_{2}+\mathcal{V}_{1}+\mathcal{V}_{4}-i \mathcal{V}_{3}\right)$ \\
\hline (1) & + & 3 & $y$ & $\uparrow$ & $\frac{1}{4}(i+\sqrt{3})\left(\mathcal{V}_{2}-\mathcal{V}_{1}-\mathcal{V}_{4}+\mathcal{V}_{3}\right)$ \\
\hline (1) & + & 3 & $y$ & $\downarrow$ & $\frac{1}{4} i(i+\sqrt{3})\left(\mathcal{V}_{2}+\mathcal{V}_{1}-\mathcal{V}_{4}-\mathcal{V}_{3}\right)$ \\
\hline (1) & + & 3 & $z$ & $\uparrow$ & $-\mathcal{V}_{4}$ \\
\hline (1) & + & 3 & $z$ & $\downarrow$ & $\mathcal{V}_{3}$ \\
\hline (1) & + & 4 & $x$ & $\uparrow$ & $\frac{1}{4}(i \sqrt{3}+1)\left(\mathcal{V}_{2}-i \mathcal{V}_{1}+i \mathcal{V}_{4}+\mathcal{V}_{3}\right)$ \\
\hline (1) & + & 4 & $x$ & $\downarrow$ & $\frac{1}{4}(i \sqrt{3}+1)\left(\mathcal{V}_{2}+i\left(\mathcal{V}_{1}+\mathcal{V}_{4}+i \mathcal{V}_{3}\right)\right)$ \\
\hline (1) & + & 4 & $y$ & $\uparrow$ & $\frac{1}{4}(i+\sqrt{3})\left(\mathcal{V}_{2}+\mathcal{V}_{1}+\mathcal{V}_{4}+\mathcal{V}_{3}\right)$ \\
\hline (1) & + & 4 & $y$ & $\downarrow$ & $\frac{1}{4} i(i+\sqrt{3})\left(-\mathcal{V}_{2}+\mathcal{V}_{1}-\mathcal{V}_{4}+\mathcal{V}_{3}\right)$ \\
\hline (1) & + & 4 & $z$ & $\uparrow$ & $-\mathcal{V}_{1}$ \\
\hline (1) & + & 4 & $z$ & $\downarrow$ & $\mathcal{V}_{2}$ \\
\hline (1) & - & 1 & $x$ & $\uparrow$ & $\frac{1}{4}(i \sqrt{3}+1)\left(\mathcal{V}_{2}-i\left(\mathcal{V}_{1}+\mathcal{V}_{4}-i \mathcal{V}_{3}\right)\right)$ \\
\hline (1) & - & 1 & $x$ & $\downarrow$ & $\frac{1}{4}(-i+\sqrt{3})\left(-i \mathcal{V}_{2}+\mathcal{V}_{1}-\mathcal{V}_{4}-i \mathcal{V}_{3}\right)$ \\
\hline (1) & - & 1 & $y$ & $\uparrow$ & $\frac{1}{4}(1-i \sqrt{3})\left(\mathcal{V}_{2}+\mathcal{V}_{1}-\mathcal{V}_{4}-\mathcal{V}_{3}\right)$ \\
\hline (1) & - & 1 & $y$ & $\downarrow$ & $\frac{1}{4}(i+\sqrt{3})\left(\mathcal{V}_{2}-\mathcal{V}_{1}-\mathcal{V}_{4}+\mathcal{V}_{3}\right)$ \\
\hline (1) & - & 1 & $z$ & $\uparrow$ & $V_{3}$ \\
\hline (1) & - & 1 & $z$ & $\downarrow$ & $\mathcal{V}_{4}$ \\
\hline (1) & - & 2 & $x$ & $\uparrow$ & $-\frac{1}{4}(-i+\sqrt{3})\left(-i \mathcal{V}_{2}+\mathcal{V}_{1}+\mathcal{V}_{4}+i \mathcal{V}_{3}\right)$ \\
\hline (1) & - & 2 & $x$ & $\downarrow$ & $\frac{1}{4}(-i+\sqrt{3})\left(-i \mathcal{V}_{2}-\mathcal{V}_{1}+\mathcal{V}_{4}-i \mathcal{V}_{3}\right)$ \\
\hline (1) & - & 2 & $y$ & $\uparrow$ & $\frac{1}{4} i(i+\sqrt{3})\left(\mathcal{V}_{2}-\mathcal{V}_{1}+\mathcal{V}_{4}-\mathcal{V}_{3}\right)$ \\
\hline (1) & - & 2 & $y$ & $\downarrow$ & $\frac{1}{4}(i+\sqrt{3})\left(\mathcal{V}_{2}+\mathcal{V}_{1}+\mathcal{V}_{4}+\mathcal{V}_{3}\right)$ \\
\hline (1) & - & 2 & $z$ & $\uparrow$ & $\mathcal{V}_{2}$ \\
\hline (1) & - & 2 & $z$ & $\downarrow$ & $\mathcal{V}_{1}$ \\
\hline (1) & - & 3 & $x$ & $\uparrow$ & $\frac{1}{4}(-i+\sqrt{3})\left(-i \mathcal{V}_{2}+\mathcal{V}_{1}+\mathcal{V}_{4}+i \mathcal{V}_{3}\right)$ \\
\hline (1) & - & 3 & $x$ & $\downarrow$ & $\frac{1}{4}(-i+\sqrt{3})\left(-i \mathcal{V}_{2}-\mathcal{V}_{1}+\mathcal{V}_{4}-i \mathcal{V}_{3}\right)$ \\
\hline (1) & - & 3 & $y$ & $\uparrow$ & $\frac{1}{4}(1-i \sqrt{3})\left(\mathcal{V}_{2}-\mathcal{V}_{1}+\mathcal{V}_{4}-\mathcal{V}_{3}\right)$ \\
\hline (1) & - & 3 & $y$ & $\downarrow$ & $\frac{1}{4}(i+\sqrt{3})\left(\mathcal{V}_{2}+\mathcal{V}_{1}+\mathcal{V}_{4}+\mathcal{V}_{3}\right)$ \\
\hline (1) & - & 3 & $z$ & $\uparrow$ & $\mathcal{V}_{2}$ \\
\hline (1) & - & 3 & $z$ & $\downarrow$ & $-\mathcal{V}_{1}$ \\
\hline
\end{tabular}


TABLE III. (Continued.)

\begin{tabular}{|c|c|c|c|c|c|}
\hline$f^{2}$ State: $\Delta$ & $f^{1}$ State: $\omega$ & Site $j$ & Atomic orbital: $\mathcal{P}$ & $c \operatorname{spin}, \sigma$ & $V_{\Delta \omega j \mathcal{P} \sigma}$ \\
\hline (1) & - & 4 & $x$ & $\uparrow$ & $-\frac{1}{4} i(-i+\sqrt{3})\left(\mathcal{V}_{2}-i\left(\mathcal{V}_{1}+\mathcal{V}_{4}-i \mathcal{V}_{3}\right)\right)$ \\
\hline (1) & - & 4 & $x$ & $\downarrow$ & $\frac{1}{4}(-i+\sqrt{3})\left(-i \mathcal{V}_{2}+\mathcal{V}_{1}-\mathcal{V}_{4}-i \mathcal{V}_{3}\right)$ \\
\hline (1) & - & 4 & $y$ & $\uparrow$ & $\frac{1}{4} i(i+\sqrt{3})\left(\mathcal{V}_{2}+\mathcal{V}_{1}-\mathcal{V}_{4}-\mathcal{V}_{3}\right)$ \\
\hline (1) & - & 4 & $y$ & $\downarrow$ & $\frac{1}{4}(i+\sqrt{3})\left(\mathcal{V}_{2}-\mathcal{V}_{1}-\mathcal{V}_{4}+\mathcal{V}_{3}\right)$ \\
\hline (1) & - & 4 & $z$ & $\uparrow$ & $V_{3}$ \\
\hline (1) & - & 4 & $z$ & $\downarrow$ & $-\mathcal{V}_{4}$ \\
\hline (2) & + & 1 & $x$ & $\uparrow$ & $\frac{1}{4}(i+\sqrt{3})\left(\mathcal{V}_{2}-\mathcal{V}_{1}-\mathcal{V}_{4}+\mathcal{V}_{3}\right)$ \\
\hline (2) & + & 1 & $x$ & $\downarrow$ & $\frac{1}{4}(i+\sqrt{3})\left(\mathcal{V}_{2}+\mathcal{V}_{1}-\mathcal{V}_{4}-\mathcal{V}_{3}\right)$ \\
\hline (2) & + & 1 & $y$ & $\uparrow$ & $-\frac{1}{4}(-i+\sqrt{3})\left(i \mathcal{V}_{2}-\mathcal{V}_{1}+\mathcal{V}_{4}+i \mathcal{V}_{3}\right)$ \\
\hline (2) & + & 1 & $y$ & $\downarrow$ & $-\frac{1}{4}(-i+\sqrt{3})\left(\mathcal{V}_{2}-i\left(\mathcal{V}_{1}+\mathcal{V}_{4}-i \mathcal{V}_{3}\right)\right)$ \\
\hline (2) & + & 1 & $z$ & $\uparrow$ & $\mathcal{V}_{4}$ \\
\hline (2) & + & 1 & $z$ & $\downarrow$ & $i \mathcal{V}_{3}$ \\
\hline (2) & + & 2 & $x$ & $\uparrow$ & $\frac{1}{4}(i+\sqrt{3})\left(\mathcal{V}_{2}+\mathcal{V}_{1}+\mathcal{V}_{4}+\mathcal{V}_{3}\right)$ \\
\hline (2) & + & 2 & $x$ & $\downarrow$ & $\frac{i}{4}(i+\sqrt{3})\left(\mathcal{V}_{2}-\mathcal{V}_{1}+\mathcal{V}_{4}-\mathcal{V}_{3}\right)$ \\
\hline (2) & + & 2 & $y$ & $\uparrow$ & $-\frac{1}{4}(i \sqrt{3}+1)\left(\mathcal{V}_{2}-i \mathcal{V}_{1}+i \mathcal{V}_{4}+\mathcal{V}_{3}\right)$ \\
\hline (2) & + & 2 & $y$ & $\downarrow$ & $\frac{1}{4}(-i+\sqrt{3})\left(\mathcal{V}_{2}+i\left(\mathcal{V}_{1}+\mathcal{V}_{4}+i \mathcal{V}_{3}\right)\right)$ \\
\hline (2) & + & 2 & $z$ & $\uparrow$ & $-\mathcal{V}_{1}$ \\
\hline (2) & + & 2 & $z$ & $\downarrow$ & $i \mathcal{V}_{2}$ \\
\hline (2) & + & 3 & $x$ & $\uparrow$ & $\frac{1}{4}(i+\sqrt{3})\left(\mathcal{V}_{2}+\mathcal{V}_{1}+\mathcal{V}_{4}+\mathcal{V}_{3}\right)$ \\
\hline (2) & + & 3 & $x$ & $\downarrow$ & $\frac{1}{4}(i+\sqrt{3})\left(-\mathcal{V}_{2}+\mathcal{V}_{1}-\mathcal{V}_{4}+\mathcal{V}_{3}\right)$ \\
\hline (2) & + & 3 & $y$ & $\uparrow$ & $-\frac{1}{4}(-i+\sqrt{3})\left(i \mathcal{V}_{2}+\mathcal{V}_{1}-\mathcal{V}_{4}+i \mathcal{V}_{3}\right)$ \\
\hline (2) & + & 3 & $y$ & $\downarrow$ & $-\frac{1}{4}(-i+\sqrt{3})\left(\mathcal{V}_{2}+i\left(\mathcal{V}_{1}+\mathcal{V}_{4}+i \mathcal{V}_{3}\right)\right)$ \\
\hline (2) & + & 3 & $z$ & $\uparrow$ & $\mathcal{V}_{1}$ \\
\hline (2) & + & 3 & $z$ & $\downarrow$ & $i \mathcal{V}_{2}$ \\
\hline (2) & + & 4 & $x$ & $\uparrow$ & $\frac{1}{4}(i+\sqrt{3})\left(\mathcal{V}_{2}-\mathcal{V}_{1}-\mathcal{V}_{4}+\mathcal{V}_{3}\right)$ \\
\hline (2) & + & 4 & $x$ & $\downarrow$ & $-\frac{1}{4}(i+\sqrt{3})\left(\mathcal{V}_{2}+\mathcal{V}_{1}-\mathcal{V}_{4}-\mathcal{V}_{3}\right)$ \\
\hline (2) & + & 4 & $y$ & $\uparrow$ & $-\frac{1}{4}(-i+\sqrt{3})\left(i \mathcal{V}_{2}-\mathcal{V}_{1}+\mathcal{V}_{4}+i \mathcal{V}_{3}\right)$ \\
\hline (2) & + & 4 & $y$ & $\downarrow$ & $\frac{1}{4}(-i+\sqrt{3})\left(\mathcal{V}_{2}-i\left(\mathcal{V}_{1}+\mathcal{V}_{4}-i \mathcal{V}_{3}\right)\right)$ \\
\hline (2) & + & 4 & $z$ & $\uparrow$ & $-\mathcal{V}_{4}$ \\
\hline (2) & + & 4 & $z$ & $\downarrow$ & $i \mathcal{V}_{3}$ \\
\hline (2) & - & 1 & $x$ & $\uparrow$ & $-\frac{1}{4}(i+\sqrt{3})\left(\mathcal{V}_{2}-\mathcal{V}_{1}+\mathcal{V}_{4}-\mathcal{V}_{3}\right)$ \\
\hline (2) & - & 1 & $x$ & $\downarrow$ & $-\frac{1}{4}(i+\sqrt{3})\left(\mathcal{V}_{2}+\mathcal{V}_{1}+\mathcal{V}_{4}+\mathcal{V}_{3}\right)$ \\
\hline (2) & - & 1 & $y$ & $\uparrow$ & $\frac{1}{4}(-i+\sqrt{3})\left(\mathcal{V}_{2}+i\left(\mathcal{V}_{1}+\mathcal{V}_{4}+i \mathcal{V}_{3}\right)\right)$ \\
\hline (2) & - & 1 & $y$ & $\downarrow$ & $-\frac{1}{4}(-i+\sqrt{3})\left(i \mathcal{V}_{2}+\mathcal{V}_{1}-\mathcal{V}_{4}+i \mathcal{V}_{3}\right)$ \\
\hline (2) & - & 1 & $z$ & $\uparrow$ & $i \mathcal{V}_{2}$ \\
\hline (2) & - & 1 & $z$ & $\downarrow$ & $-\mathcal{V}_{1}$ \\
\hline (2) & - & 2 & $x$ & $\uparrow$ & $-\frac{1}{4}(i+\sqrt{3})\left(\mathcal{V}_{2}+\mathcal{V}_{1}-\mathcal{V}_{4}-\mathcal{V}_{3}\right)$ \\
\hline (2) & - & 2 & $x$ & $\downarrow$ & $-\frac{1}{4}(i+\sqrt{3})\left(\mathcal{V}_{2}-\mathcal{V}_{1}-\mathcal{V}_{4}+\mathcal{V}_{3}\right)$ \\
\hline (2) & - & 2 & $y$ & $\uparrow$ & $-\frac{1}{4}(-i+\sqrt{3})\left(\mathcal{V}_{2}-i\left(\mathcal{V}_{1}+\mathcal{V}_{4}-i \mathcal{V}_{3}\right)\right)$ \\
\hline (2) & - & 2 & $y$ & $\downarrow$ & $\frac{1}{4}(-i+\sqrt{3})\left(-i \mathcal{V}_{2}+\mathcal{V}_{1}-\mathcal{V}_{4}-i \mathcal{V}_{3}\right)$ \\
\hline (2) & - & 2 & $z$ & $\uparrow$ & $i \mathcal{V}_{3}$ \\
\hline (2) & - & 2 & $z$ & $\downarrow$ & $\mathcal{V}_{4}$ \\
\hline (2) & - & 3 & $x$ & $\uparrow$ & $\frac{1}{4}(i+\sqrt{3})\left(\mathcal{V}_{2}+\mathcal{V}_{1}-\mathcal{V}_{4}-\mathcal{V}_{3}\right)$ \\
\hline (2) & - & 3 & $x$ & $\downarrow$ & $\frac{1}{4}(i+\sqrt{3})\left(-\mathcal{V}_{2}+\mathcal{V}_{1}+\mathcal{V}_{4}-\mathcal{V}_{3}\right)$ \\
\hline (2) & - & 3 & $y$ & $\uparrow$ & $\frac{1}{4}(-i+\sqrt{3})\left(\mathcal{V}_{2}-i\left(\mathcal{V}_{1}+\mathcal{V}_{4}-i \mathcal{V}_{3}\right)\right)$ \\
\hline (2) & - & 3 & $y$ & $\downarrow$ & $\frac{i}{4}(-i+\sqrt{3})\left(-i \mathcal{V}_{2}+\mathcal{V}_{1}-\mathcal{V}_{4}-i \mathcal{V}_{3}\right)$ \\
\hline (2) & - & 3 & $z$ & $\uparrow$ & $i \mathcal{V}_{3}$ \\
\hline (2) & - & 3 & $z$ & $\downarrow$ & $-\mathcal{V}_{4}$ \\
\hline (2) & - & 4 & $x$ & $\uparrow$ & $\frac{1}{4}(i+\sqrt{3})\left(\mathcal{V}_{2}-\mathcal{V}_{1}+\mathcal{V}_{4}-\mathcal{V}_{3}\right)$ \\
\hline (2) & - & 4 & $x$ & $\downarrow$ & $-\frac{1}{4}(i+\sqrt{3})\left(\mathcal{V}_{2}+\mathcal{V}_{1}+\mathcal{V}_{4}+\mathcal{V}_{3}\right)$ \\
\hline (2) & - & 4 & $y$ & $\uparrow$ & $-\frac{1}{4}(-i+\sqrt{3})\left(\mathcal{V}_{2}+i\left(\mathcal{V}_{1}+\mathcal{V}_{4}+i \mathcal{V}_{3}\right)\right)$ \\
\hline (2) & - & 4 & $y$ & $\downarrow$ & $\frac{1}{4}(-i+\sqrt{3})\left(-i \mathcal{V}_{2}-\mathcal{V}_{1}+\mathcal{V}_{4}-i \mathcal{V}_{3}\right)$ \\
\hline (2) & - & 4 & $z$ & $\uparrow$ & $i \mathcal{V}_{2}$ \\
\hline (2) & - & 4 & $z$ & $\downarrow$ & $\mathcal{V}_{1}$ \\
\hline
\end{tabular}


izing the parameters of the Hamiltonian, and as such can be thought of as a renormalization procedure. Since this a standard second-order canonical transformation [6,50], we neglect the step-by-step procedure, and rather choose to highlight the key steps and results that make it different from its usual implementation in the original Anderson model.

The Hamiltonian is split into two pieces: (i) $H_{0}$ which contains the terms acting independently on the low-energy $\left(f^{2}\right)$ and high-energy $\left(f^{1}\right)$ subspaces and (ii) $H_{1}$ which connects the low-energy and high-energy subspaces, i.e., $H=H_{0}+H_{1}$, where

$$
H_{0}=\sum_{\alpha, \sigma} \epsilon_{\alpha} c_{\alpha \sigma}^{\dagger} c_{\alpha \sigma}+\epsilon_{f} \sum_{\Delta}\left|f^{2}, \Delta\right\rangle\left\langle f^{2}, \Delta\right|,
$$

where $\alpha$ sums over the molecular orbitals, $\sigma$ is the spin of the conduction electrons, $\Delta$ sums over the two $f^{2}$ non-Kramers ground states, and $\epsilon_{f}$ is the energy level spacing between the $f^{2}$ ground state and the excited $f^{1}$ state. The $f^{1}$ state is placed at zero-energy so as to ignore its presence in $H_{0}$. The use of molecular orbitals, instead of the atomic orbitals on each site, is beneficial as it renders the conduction electron kinetic term diagonal; the tight-binding hopping matrix on the single tetrahedron has molecular orbitals (eigenstates) decoupled as $A_{1} \oplus E \oplus T_{1} \oplus 2 T_{2}$, where we use the $T_{d}$ irrep notation. Here, $E$ and $T_{2}$ have molecular $e_{g}$ and $p$ as its basis functions.

The connecting-to the high energy space term is

$$
H_{1}=\sum_{\Delta, \omega, \alpha, \sigma} \tilde{V}_{\Delta \omega \alpha \sigma}\left|f^{2}, \Delta\right\rangle\left\langle f^{1}, \omega\right| c_{\alpha \sigma}+\text { H.c. }
$$

where $\tilde{V}_{\Delta \omega \alpha \sigma}$ involves linear combinations of the atomic hybridization matrix elements presented in Table III, $\omega$ sums over the $f^{1}$ states, and we again employ the conduction electron molecular orbitals, $\alpha$. Due to the Hubbard operators not obeying standard fermion/boson commutation rules, it is cumbersome to employ them directly in the SW transformation. Instead, we employ the pseudoparticle method [51],

$$
b_{\omega}^{\dagger}|0\rangle=\left|f^{1}, \omega\right\rangle, \quad f_{\Delta}^{\dagger}|0\rangle=\left|f^{2}, \Delta\right\rangle,
$$

where $|0\rangle$ is the vacuum, $b_{\omega}^{\dagger}$ is a pseudoboson operator that creates the $\left|f^{1}, \omega\right\rangle$ state, and $f_{\Delta}^{\dagger}$ is a pseudofermion operator that creates the $\left|f^{2}, \Delta\right\rangle$ state. Indeed, one can interchange the usage of a pseudoboson and pseudofermion for the two configurations, as we must only ensure that one configuration is fermionic while the other is bosonic, so as to retain the overall fermionic structure of hybridization. We can then rewrite the hybridization term as

$$
H_{1}=\sum_{\Delta, \omega, \alpha, \sigma} \tilde{V}_{\Delta \omega \alpha \sigma} f_{\Delta}^{\dagger} b_{\omega} c_{\alpha \sigma}+\text { H.c. }
$$

We now apply the SW transformation, i.e., $H_{\text {eff }}=\mathrm{e}^{S}\left(H_{0}+\right.$ $\left.H_{1}\right) \mathrm{e}^{-S}=H_{0}+\frac{1}{2}\left[S, H_{1}\right]$. We find that the generator of the transformation, $S$, is

$$
S=\sum_{\Delta, \omega, \alpha, \sigma} \frac{\tilde{V}_{\Delta \omega \alpha \sigma}}{\epsilon_{f}-\epsilon_{\alpha}} f_{\Delta}^{\dagger} b_{\omega} c_{\alpha \sigma}-\text { H.c. }
$$

$$
\begin{aligned}
& \mathcal{G}_{c}\left(i \omega_{n}, \vec{k}\right) \equiv \longrightarrow=\frac{1}{i \omega_{n}-\epsilon_{k}} \\
& \mathcal{G}_{f}\left(i \omega_{n}\right) \equiv--=\frac{1}{i \omega_{n}-\lambda}
\end{aligned}
$$

FIG. 3. Green's function for conduction (solid line) and $f$ (dashed line) electrons.

Working through the commutators, we arrive at the effective low-energy Hamiltonian,

$$
H_{\mathrm{eff}}=-\frac{1}{2} \sum_{\Delta, \Delta^{\prime}, \omega, \alpha, \alpha^{\prime}, \sigma, \sigma^{\prime}} \frac{\tilde{V}_{\Delta \omega \alpha \sigma} \tilde{V}_{\Delta^{\prime} \omega \alpha^{\prime} \sigma^{\prime}}^{*}}{\epsilon_{f}-\epsilon_{\alpha}} f_{\Delta}^{\dagger} f_{\Delta^{\prime}} c_{\alpha^{\prime} \sigma^{\prime}}^{\dagger} c_{\alpha \sigma}+\text { H.c. }
$$

For clarity, we note that the SW transformation generates terms of the form $b_{\omega}^{\dagger} b_{\omega^{\prime}} F\left(f, f^{\dagger}, c, c^{\dagger}\right)$, where $F(\ldots)$ is a quartic function in the $f$ and $c$ operators; however, in the low-energy subspace, the $f^{1}$ state is unoccupied and so these terms vanish in the low-energy theory. Equation (F6) leads to the Kondo Hamiltonians presented in the main text, which were based on symmetry analysis.

\section{APPENDIX G: PERTURBATIVE RENORMALIZATION GROUP APPROACH FOR MULTIPOLAR QUANTUM IMPURITY MODELS}

In this section, we discuss the general strategy used to tackle multipolar quantum impurity systems and obtain the renormalization group (RG) $\beta$ function for the various coupling constants. Specifically, we are interested in computing the scattering amplitude which is obtained by perturbatively computing the four-point correlation function, $\Gamma$.

We consider a general Hamiltonian of the system, without specifying the particular orbitals, as

$H=\sum_{k, a, \alpha} \epsilon_{k} c_{k, a, \alpha}^{\dagger} c_{k, a, \alpha}+H_{\text {Kondo }}\left[c^{\dagger}, c, \vec{S}\right]$, where $a$ and $\alpha$ refer to the orbital and spin index of the conduction electron. $H_{\text {Kondo }}\left[c^{\dagger}, c, \vec{S}\right]$ denotes the Kondo interaction and is quartic in fermionic operators; the specific form depends on the orbitals of interest. We take the conduction electron bands to be degenerate, and we make the further simplification (in the spirit of Anderson [52]) of a constant density of states: $\rho(E)=\rho$, where $-D<E<D$ is the energy window and $D$ is the bandwidth. We note that choosing different density of states for the different orbitals does not modify the subsequent RG flow equations. The Fermi level is taken as the zeroenergy level. We also represent the pseudospin- $1 / 2$ operator in terms of Abrikosov pseudofermions, $\vec{S}=\frac{1}{2} \sum_{\mu= \pm} f_{\mu}^{\dagger} \vec{\sigma}_{\mu, \nu} f_{\nu}$, where $f$ denote the pseudofermions. As is typical when using Abrikosov pseudofermions, the Hilbert space gets enlarged by their introduction through inclusion of unphysical doubly occupied and unoccupied states. To correct for this, we employ the standard Popov-Fedotov trick of introducing a complex chemical potential $\left(\lambda=\frac{i \pi}{2 \beta}\right)$ to restrict the Hilbert space; the complex chemical potential ensures that the partition function contributions from the two unphysical sectors perfectly cancel each other. We note that we define the Green's function (along with their diagrammatic propagators) for the conduction electron and localized $f$ electron in Fig. 3. 


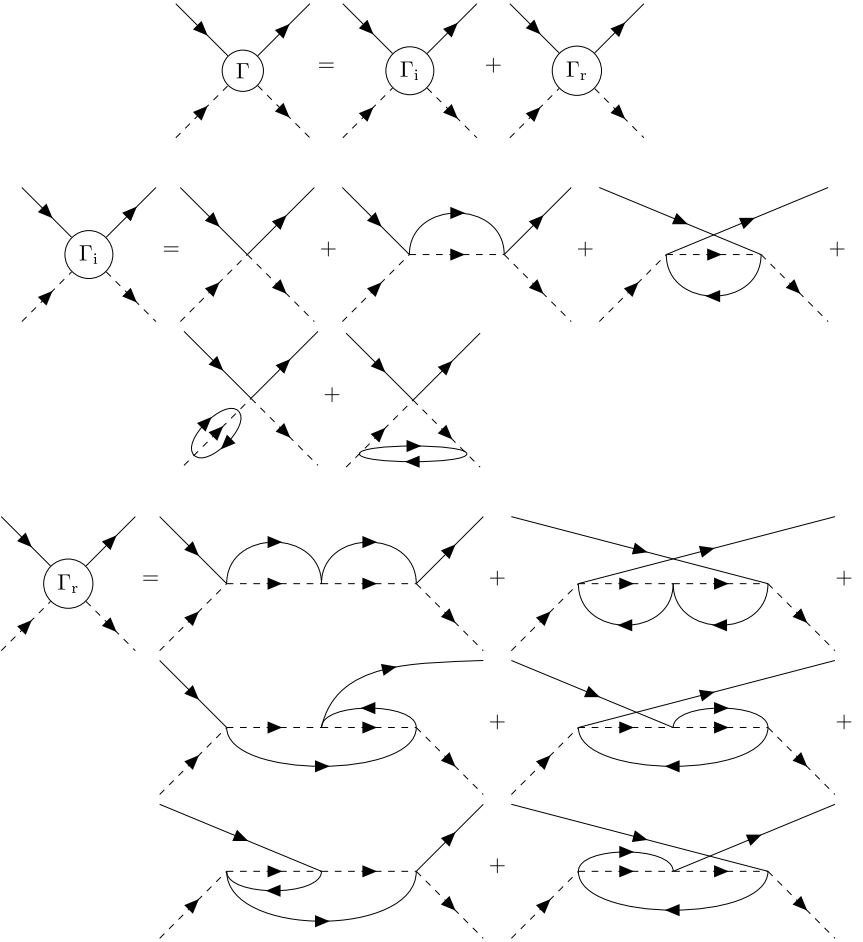

FIG. 4. Four-point function $(\Gamma)$ which is composed of twoparticle irreducible $\left(\Gamma_{\mathrm{i}}\right)$ and reducible $\left(\Gamma_{\mathrm{r}}\right)$ diagrams. $\Gamma_{\mathrm{i}}$ contains the logarithmically divergent terms necessary for the computation of the $\beta$ function.

We are now at the stage to compute the four-point correlation function $(\Gamma)$. We present the four point function up to two-loop (or third order in the Kondo interaction strength) in Fig. 4, where we have organized the diagrams into $\Gamma_{i}$ $\left(\Gamma_{r}\right)$ to categorize diagrams that are two-particle irreducible (reducible). We compute the vertex function in the limit of

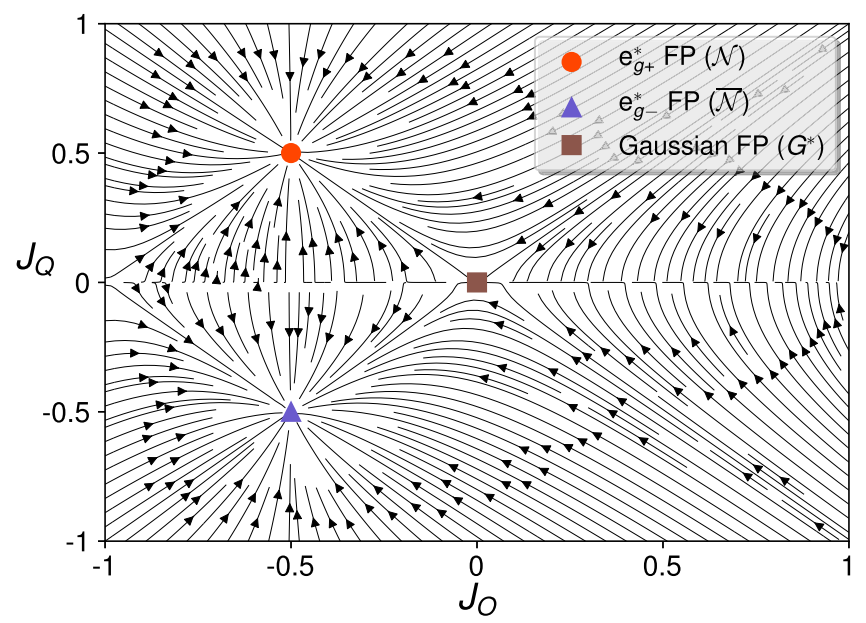

FIG. 5. RG flow diagram of $e_{g}$-only model in the $J_{O}-J_{Q}$ plane. The Nozières fixed points $\left(e_{g} \pm^{*}\right)$ in lines $\mathcal{N}$ and $\overline{\mathcal{N}}$ are denoted by the red dot and blue triangles, respectively. The Gaussian fixed point is denoted by the brown square. The Ising-like line of fixed points, with regions where it is repulsive $\left(J_{O}<0\right)$ and attractive $\left(J_{O}>0\right)$ is apparent.

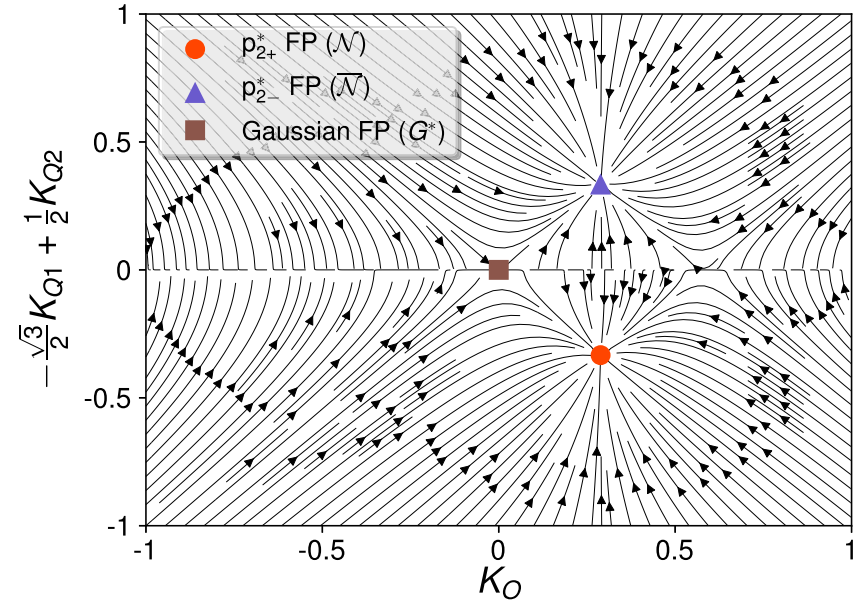

FIG. 6. RG flow diagram of $p$-orbital-only model in a particular 2D plane spanned by $-\frac{\sqrt{3}}{2} K_{Q 1}+\frac{1}{2} K_{Q 2}$ and $K_{O}$. The $p$-only fixed points $\left(p_{2 \mp}^{*}\right)$ in lines $\mathcal{N}$ and $\overline{\mathcal{N}}$ are denoted by the red dot and blue triangles, respectively. The Gaussian fixed point is denoted by the brown square. The Ising-like line of fixed points is apparent.

$v \ll D$ (where $v$ is the energy sum of the incoming conduction and $f$ electrons) and retain all the leading logarithmically divergent terms. It is the irreducible diagrams that provide the logarithmically divergent terms. Finally, we apply the Wilsonian RG procedure: the low-energy scattering amplitude/rate is independent of the UV cutoff (D), i.e., $\rho \Gamma(\nu, D, \vec{g})=$ $\rho \Gamma\left(\nu, D^{\prime}, \vec{g}\left(D^{\prime}\right)\right)$, where $\vec{g}$ and $\vec{g}\left(D^{\prime}\right)$ symbolically represent the bare and renormalized coupling constants. Solving for the renormalized coupling constant yields the desired $\beta$ function. For completeness, we note that under the RG, symmetry allowed terms of the form $\sigma_{\alpha \beta}^{0,1,2,3} c_{x, \alpha}^{\dagger} c_{y, \beta}$ are also generated. Importantly, these terms only introduce/renormalize hopping amplitudes, and not the multipolar Kondo couplings, and as such do not affect the obtained $\beta$ functions.

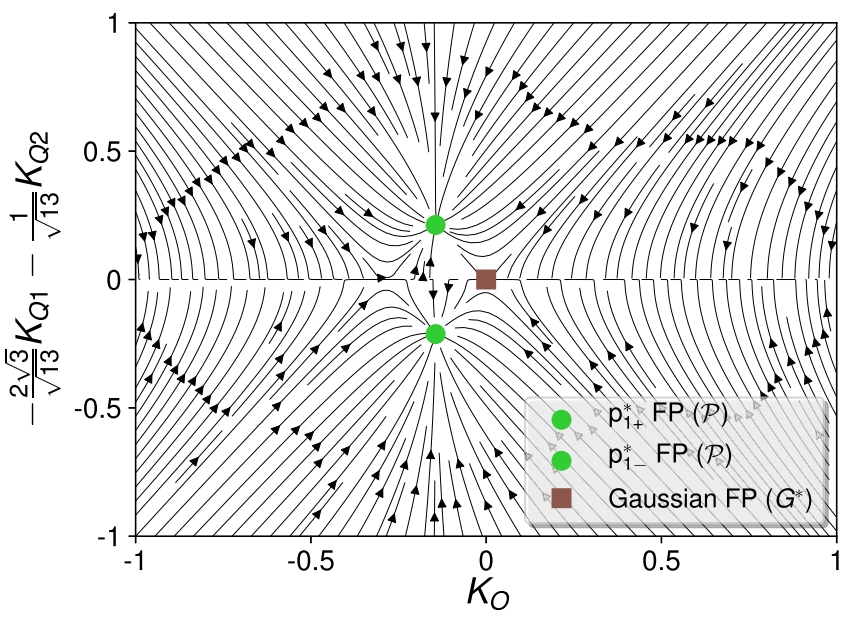

FIG. 7. RG flow diagram of $p$-orbital-only model in a particular 2D plane spanned by $-\frac{2 \sqrt{3}}{\sqrt{13}} K_{Q 1}-\frac{1}{\sqrt{13}} K_{Q 2}$ and $K_{O}$. The $p$-only fixed points $\left(p_{1 \mp}^{*}\right.$ ) in lines $\mathcal{P}$ are denoted by green dots. The Gaussian fixed point is denoted by the brown square. The Ising-like line of fixed points is apparent. 


\section{APPENDIX H: RENORMALIZATION GROUP FLOW DIAGRAMS OF $e_{g}$ AND $p$ FIXED POINTS}

In Figs. 5, 6, and 7, we depict the RG flow diagrams for the $e_{g}$ only model, and for the $p$ model (for the plane including the $p_{1 \pm}^{*}$ and $p_{2 \pm}^{*}$ fixed points). Due to the similarity of the
RG flow diagrams in Figs. 5 and 6 about the respective pair of fixed points, it is suggestive that they describe similar low-energy physics. The RG flow diagram depicted in Fig. 7 is qualitatively distinct in terms of flow nearby to the fixed points.

\section{APPENDIX I: HAMILTONIAN AND $\beta$ FUNCTIONS: INCLUSION OF $e_{g} \otimes p$ MIXING}

We present here the Kondo couplings that mix the $e_{g}$ and $p$ orbitals. First, we present the Kondo Hamiltonians that introduce the three additional couplings: quadrupolar $L_{Q 1}, L_{Q 2}$ and octupolar $L_{O}$.

$$
\begin{gathered}
H_{O}^{e_{g} \otimes p}=L_{O} S_{j}^{z} c_{j, a, \alpha}^{\dagger}\left[\sigma_{\alpha \beta}^{x} \otimes\left(-\sqrt{3} \kappa_{a b}^{2 x, r}+\kappa_{a b}^{1 x, r}\right)+\sigma_{\alpha \beta}^{y} \otimes\left(\sqrt{3} \kappa_{a b}^{2 y, r}+\kappa_{a b}^{1 y, r}\right)-2 \sigma_{\alpha \beta}^{z} \otimes \kappa_{a b}^{1 z, r}\right] c_{j, b, \beta}, \\
H_{Q 1}^{e_{g} \otimes p}=-L_{Q 1} c_{j, a, \alpha}^{\dagger}\left[\left(\frac{\sqrt{3}}{2} S_{j}^{x}\right) \sigma_{\alpha \beta}^{x} \otimes \kappa_{a b}^{2 x, i}+\left(S_{j}^{x}-\frac{\sqrt{3}}{2} S_{j}^{y}\right) \sigma_{\alpha \beta}^{x} \otimes \kappa_{a b}^{1 x, i}\right. \\
\left.-\left(\frac{\sqrt{3}}{2} S_{j}^{x}\right) \sigma_{\alpha \beta}^{y} \otimes \kappa_{a b}^{2 y, i}+\left(S_{j}^{x}+\frac{\sqrt{3}}{2} S_{j}^{y}\right) \sigma_{\alpha \beta}^{y} \otimes \kappa_{a b}^{1 y, i}-\left(\frac{3}{2} S_{j}^{y}\right) \sigma_{\alpha \beta}^{z} \otimes \kappa_{a b}^{2 z, i}-\left(\frac{S_{j}^{x}}{2}\right) \sigma_{\alpha \beta}^{z} \otimes \kappa_{a b}^{1 z, i}\right] c_{j, b, \beta}, \\
H_{Q 2}^{e_{g} \otimes p}=-L_{Q 2} c_{j, a, \alpha}^{\dagger}\left[\left(\frac{\sqrt{3}}{2} S_{j}^{x}+S_{j}^{y}\right) \sigma_{\alpha \beta}^{x} \otimes \kappa_{a b}^{2 x, i}-\left(\frac{\sqrt{3}}{2} S_{j}^{y}\right) \sigma_{\alpha \beta}^{x} \otimes \kappa_{a b}^{1 x, i}+\left(-\frac{\sqrt{3}}{2} S_{j}^{x}+S_{j}^{y}\right) \sigma_{\alpha \beta}^{y} \otimes \kappa_{a b}^{2 y, i}\right. \\
\left.+\left(\frac{\sqrt{3}}{2} S_{j}^{y}\right) \sigma_{\alpha \beta}^{y} \otimes \kappa_{a b}^{1 y, i}-\left(\frac{S_{j}^{y}}{2}\right) \sigma_{\alpha \beta}^{z} \otimes \kappa_{a b}^{2 z, i}-\left(\frac{3}{2} S_{j}^{x}\right) \sigma_{\alpha \beta}^{z} \otimes \kappa_{a b}^{1 z, i}\right] c_{j, b, \beta},
\end{gathered}
$$

where $\kappa$ are the generalized SU(5) Gell-Mann Matrices describing the orbital degree of freedom $\left\{x^{2}-y^{2}, 2 z^{2}-x^{2}-y^{2}\right\} \otimes$ $\{x, y, z\}$ as listed in SI D.

The resulting complete RG flow equations are

$$
\begin{aligned}
& \frac{d K_{Q 1}}{d \ln D}=6 K_{Q 2} K_{O}-\sqrt{3} L_{O}\left(L_{Q 1}+3 L_{Q 2}\right) \\
& +K_{Q 1}\left(12 K_{Q 2}^{2}+6 K_{O}^{2}+2 K_{Q 1}^{2}+24 L_{O}^{2}+2 J_{Q}^{2}+\frac{15}{2} L_{Q 1}^{2}+9 L_{Q 1} L_{Q 2}+\frac{15}{2} L_{Q 2}^{2}+2 J_{O}^{2}\right), \\
& \frac{d K_{Q 2}}{d \ln D}=K_{O}\left(K_{Q 1}-\sqrt{3} K_{Q 2}\right)+\frac{L_{O}}{2}\left(5 L_{Q 1}+3 L_{Q 2}\right) \\
& +K_{Q 2}\left(12 K_{Q 2}^{2}+6 K_{O}^{2}+2 K_{Q 1}^{2}+24 L_{O}^{2}+2 J_{Q}^{2}+\frac{15}{2} L_{Q 1}^{2}+9 L_{Q 1} L_{Q 2}+\frac{15}{2} L_{Q 2}^{2}+2 J_{O}^{2}\right), \\
& \frac{d K_{O}}{d \ln D}=4 K_{Q 1} K_{Q 2}-2 \sqrt{3} K_{Q 2}^{2}-\sqrt{3}\left(L_{Q 1}+L_{Q 2}\right)^{2}+K_{O}\left(24 K_{Q 2}^{2}+4 K_{Q 1}^{2}+4 J_{Q}^{2}+15 L_{Q 1}^{2}+18 L_{Q 1} L_{Q 2}+15 L_{Q 2}^{2}\right) \text {, } \\
& \frac{d J_{Q}}{d \ln D}=2 J_{Q} J_{O}-6 L_{O}\left(L_{Q 1}+L_{Q 2}\right)+J_{Q}\left(12 K_{Q 2}^{2}+6 K_{O}^{2}+2 K_{Q 1}^{2}+24 L_{O}^{2}+2 J_{Q}^{2}+\frac{15}{2} L_{Q 1}^{2}+9 L_{Q 1} L_{Q 2}+\frac{15}{2} L_{Q 2}^{2}+2 J_{O}^{2}\right) \text {, } \\
& \frac{d J_{O}}{d \ln D}=2 J_{Q}^{2}+\frac{9}{4}\left(L_{Q 1}^{2}+L_{Q 2}^{2}\right)+\frac{15}{2} L_{Q 1} L_{Q 2}+J_{O}\left(24 K_{Q 2}^{2}+4 K_{Q 1}^{2}+4 J_{Q}^{2}+15 L_{Q 1}^{2}+18 L_{Q 1} L_{Q 2}+15 L_{Q 2}^{2}\right), \\
& \frac{d L_{O}}{d \ln D}=-J_{Q}\left(L_{Q 1}+L_{Q 2}\right)-\frac{K_{Q 1}}{2 \sqrt{3}}\left(L_{Q 1}+3 L_{Q 2}\right)+\frac{K_{Q 2}}{2}\left(5 L_{Q 1}+3 L_{Q 2}\right) \\
& +L_{O}\left(24 K_{Q 2}^{2}+4 K_{Q 1}^{2}+4 J_{Q}^{2}+15 L_{Q 1}^{2}+18 L_{Q 1} L_{Q 2}+15 L_{Q 2}^{2}\right), \\
& \frac{d L_{Q 1}}{d \ln D}=L_{Q 2} J_{O}-\frac{\sqrt{3}}{2} K_{O}\left(L_{Q 1}+L_{Q 2}\right)-L_{O}\left(J_{Q}-\frac{K_{Q 1}}{\sqrt{3}}-4 K_{Q 2}\right) \\
& +L_{Q 1}\left(12 K_{Q 2}^{2}+6 K_{O}^{2}+2 K_{Q 1}^{2}+24 L_{O}^{2}+2 J_{Q}^{2}+\frac{15}{2} L_{Q 1}^{2}+9 L_{Q 1} L_{Q 2}+\frac{15}{2} L_{Q 2}^{2}+2 J_{O}^{2}\right),
\end{aligned}
$$




$$
\begin{aligned}
\frac{d L_{Q 2}}{d \ln D}= & L_{Q 1} J_{O}-\frac{\sqrt{3}}{2} K_{O}\left(L_{Q 1}+L_{Q 2}\right)-L_{O}\left(J_{Q}+\sqrt{3} K_{Q 1}\right) \\
& +L_{Q 2}\left(12 K_{Q 2}^{2}+6 K_{O}^{2}+2 K_{Q 1}^{2}+24 L_{O}^{2}+2 J_{Q}^{2}+\frac{15}{2} L_{Q 1}^{2}+9 L_{Q 1} L_{Q 2}+\frac{15}{2} L_{Q 2}^{2}+2 J_{O}^{2}\right) .
\end{aligned}
$$

The stable lines of fixed points are

Line $\mathcal{N}$ :

$$
\begin{aligned}
K_{Q 1} & =\frac{1-2 J_{Q}}{2 \sqrt{3}}, \quad K_{O}=K_{Q 1}, \quad K_{Q 2}=-\frac{K_{Q 1}}{\sqrt{3}}, \quad J_{Q} \in[0,1 / 2], \quad J_{O}=-J_{Q}, \\
L_{O} & =L_{Q 1}=L_{Q 2}= \pm \frac{\sqrt{J_{Q}\left(1-2 J_{Q}\right)}}{2 \sqrt{3}}
\end{aligned}
$$

Line $\overline{\mathcal{N}}$ :

$$
\begin{aligned}
& K_{Q 1}=-\frac{1+2 J_{Q}}{2 \sqrt{3}}, \quad K_{O}=-K_{Q 1}, \quad K_{Q 2}=-\frac{K_{Q 1}}{\sqrt{3}}, \quad J_{Q} \in[-1 / 2,0], \quad J_{O}=J_{Q}, \\
& L_{O}=-L_{Q 1}=-L_{Q 2}= \pm \frac{\sqrt{-J_{Q}\left(1+2 J_{Q}\right)}}{2 \sqrt{3}}
\end{aligned}
$$

Line $\mathcal{P}$ :

$$
\begin{aligned}
& K_{Q 1} \in\left[-\frac{1}{2 \sqrt{6}}, \frac{1}{2 \sqrt{6}}\right], \quad K_{O}=-2 \sqrt{3} K_{Q 1}^{2}, \quad K_{Q 2}=\frac{K_{Q 1}}{\sqrt{12}}, \quad J_{Q}=0, \quad J_{O}=\frac{1}{4}-6 K_{Q 1}^{2}, \\
& L_{O}= \pm \frac{K_{Q 1} \sqrt{1-24 K_{Q 1}^{2}}}{2}, \quad L_{Q 1}=-L_{Q 2}=\mp \frac{\sqrt{1-24 K_{Q 1}^{2}}}{4 \sqrt{3}} .
\end{aligned}
$$

\section{APPENDIX J: RUNNING COUPLING CONSTANT}

In studying the physical observables associated with the fixed points, it is necessary to obtain the behavior of the coupling constant in the vicinity of the fixed point. To do so, we consider general $\beta$ functions associated with the multiple general coupling constants, $\frac{d}{d \ln D} g_{l}=\beta_{l}(g)$, where $l=1,2, \ldots$ indexes the multiple couplings. In the vicinity of the fixed point, we have $g_{l} \approx g_{l}^{*}$, where $g_{l}^{*}$ is the $l^{\text {th }}$ coupling constant fixed point value. We expand the above general $\beta$ function in the neighborhood of the fixed point to obtain [53]

$$
\frac{d}{d \ln D}\left(g_{l}-g_{l}^{*}\right)=\beta_{l}\left(g-g^{*}\right) \approx \beta_{l}\left(g^{*}\right)+\sum_{k} \mathbb{M}_{k}^{l}\left(g_{k}-g_{k}^{*}\right),
$$

where $\mathbb{M}_{k}^{l}=\frac{\partial \beta_{l}}{\partial g_{k}}$ is the Jacobian matrix associated with the $\beta$ function. The first term on the right-hand side is zero, by definition of a fixed point, and we solve the subsequent matrix differential equation to obtain

$$
g_{l}(D)=g_{l}^{*}+\sum_{k} c_{k} D^{\lambda_{k}} u_{l}^{k},
$$

where $c_{k}$ are to-be-determined coefficients, and $\lambda_{k}$ and $u_{l}^{k}$ are the eigenvalues and eigenvectors, respectively, of the Jacobian matrix. Since we have obtained such an expression by examining behavior in close vicinity of the fixed point, and since the RG flow is physically controlled by tuning down the temperature, we can take (at least dimensionally) $D \sim T \ll 1$. Doing so, we obtain the temperature-dependent behavior of the coupling constant—also known as the running coupling constant-at very low temperatures (and close to the fixed point). As mentioned above, the coefficients $c_{k}$ are determined by initial conditions of the differential equation (J1), but we can go further by taking all coefficients with $\lambda_{k} \leqslant 0$ to be zero. The physical justification is that as $T \rightarrow 0$, we want the running coupling to arrive at the fixed point; if $\lambda_{k}<0 \Rightarrow T^{\lambda_{k}} \rightarrow \infty$, which corresponds to flowing away from the fixed point, and $\lambda_{k}=0 \Rightarrow T^{\lambda_{k}} \rightarrow 1$, which entails that (at this order of perturbation), the running coupling does not move from its original location in the parameter space.

\section{APPENDIX K: FREE ENERGY AND SPECIFIC HEAT}

To calculate the specific heat, we first need to compute the free energy of the system. We do so by using the linked-cluster expansion theorem

$$
F=F_{0}-\sum_{l=1}^{\infty} \frac{1}{l} F_{l},
$$

where $F_{0}$ is the free energy of the noninteracting (i.e., vanishing Kondo interaction) model, and $F_{l}$ contains all the different, connected diagrams with $l$ Kondo interactions. Diagrammatically, we can represent each term in the free energy as in Fig. 8. We note that the two diagrams of $F_{3}$ yield the same expression after computing the Matsubara sums and internal integrals. $F_{1}$ vanishes due to the internal sum over the pseudospin of a single closed loop is equivalent of taking $\operatorname{Tr}\left[S^{i}\right]=0$. To extract out the linear in $T$ behavior we need to carefully perform the Matsubara sums and energy integrals, as described below. The perturbative evaluation of 


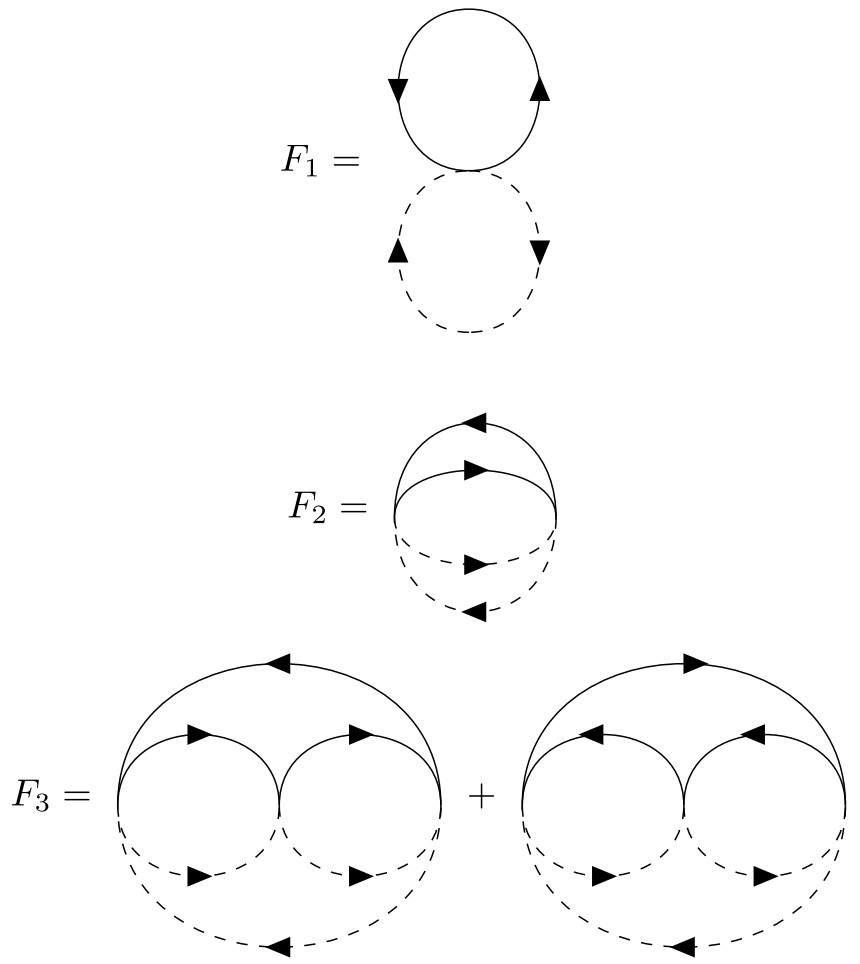

FIG. 8. Free energy diagrams up to third order in perturbation theory.

the free energy is performed using the bare couplings. Once computed, we then apply the RG procedure of taking the bare coupling constants to the running coupling constants. The specific heat is thus finally obtained by taking

$$
c_{v}=-T \frac{\partial^{2} F}{\partial T^{2}}
$$

\section{Integrals of free energy}

In order to obtain the specific heat, it is important to extract the temperature dependence in the free energy. We have both an implicit- and explicit-temperature dependence. The implicit-temperature dependence arises from the running coupling constants, as discussed above. For the models considered in this work, the explicit temperature dependence is identical, regardless of which fixed point/line we consider. Here, we provide details on obtaining the explicit temperature dependence at second and third order in perturbation theory.

First, we compute the second-order free energy diagrams. Performing the internal summation over the orbital and spin degrees of freedom and the Matsubara summations in the order of fermionic frequencies $i \omega_{3}, i \omega_{2}$ and then $i v_{1}$; this specific order avoids complications regarding the multiplicity of the Matsubara pole structure. For the second-order diagram, we obtain the following expression:

$$
F_{2}=-\frac{1}{2} \mathcal{F}_{2} \int_{-D}^{D} d \epsilon_{1} d \epsilon_{2} \frac{f\left(\epsilon_{1}\right)-f\left(\epsilon_{2}\right)}{\epsilon_{1}-\epsilon_{2}},
$$

where $\mathcal{F}_{2}=\left(4 J_{Q}^{2}+24 K_{Q 2}^{2}+6 K_{O}^{2}+4 K_{Q 1}^{2}+24 L_{O}^{2}+2 J_{O}^{2}+\right.$ $\left.18 L_{Q 1} L_{Q 2}+15 L_{Q 1}^{2}+15 L_{Q 2}^{2}\right)$. The implicit-temperature dependence mentioned above is encoded in $\mathcal{F}_{2}$. We compute this integral by first performing the integral over $\epsilon_{1}$, and then we perform a Sommerfeld expansion. Specifically, we avoid the vanishing denominator by choosing the domains of $\epsilon_{1}$ and $\epsilon_{2}$ symmetrically about the problematic domain of integration, i.e., when $\epsilon_{1}=\epsilon_{2}$. The subsequent explicit-temperature result is a constant $+T^{2}$ scaling behavior, as is commonly seen in Sommerfeld expansions.

Second, we consider the third-order free energy correction. The Matsubara summations are again performed in the order of $i \omega_{3}, i \omega_{2}$ and then $i v_{1}, i v_{2}$, to avoid the same multiplicity of pole issue described in $F_{2}$ above. After lengthy simplifications, we arrive at the following expression:

$$
\begin{aligned}
F_{3}= & \frac{1}{2} \mathcal{F}_{3} \int_{-D}^{D} d \epsilon_{1} d \epsilon_{2} d \epsilon_{3}\left(\frac{f\left(-\epsilon_{1}\right) f\left(\epsilon_{2}\right) f\left(\epsilon_{3}\right)}{\left(\epsilon_{1}-\epsilon_{2}\right)\left(\epsilon_{1}-\epsilon_{3}\right)}\right. \\
& \left.+\frac{f\left(\epsilon_{1}\right) f\left(-\epsilon_{2}\right) f\left(\epsilon_{3}\right)}{\left(\epsilon_{2}-\epsilon_{1}\right)\left(\epsilon_{2}-\epsilon_{3}\right)}+\frac{\left.f\left(\epsilon_{1}\right) f\left(\epsilon_{2}\right) f-\epsilon_{3}\right)}{\left(\epsilon_{3}-\epsilon_{1}\right)\left(\epsilon_{3}-\epsilon_{2}\right)}\right),
\end{aligned}
$$

where $\quad \mathcal{F}_{3}=\frac{3}{4}\left[48 J_{Q} L_{O}\left(L_{Q 1}+L_{Q 2}\right)-8 J_{Q}^{2} J_{O}-24 K_{Q 2}\left(2 K_{O}\right.\right.$ $\left.K_{Q 1}+L_{O}\left(5 L_{Q 1}+3 L_{Q 2}\right)\right)+24 \sqrt{3} K_{Q 2}^{2} K_{O}+8 \sqrt{3} K_{Q 1} L_{O}$ $\left(L_{Q 1}+3 L_{Q 2}\right)+24 \sqrt{3} K_{O} L_{Q 1} L_{Q 2}+12 \sqrt{3} K_{O}\left(L_{Q 1}^{2}+L_{Q 2}^{2}\right)-$ $\left.30 J_{O} L_{Q 1} L_{Q 2}-9 J_{O}\left(L_{Q 1}^{2}+L_{Q 2}^{2}\right)\right]$. The implicit-temperature dependence mentioned above is encoded in $\mathcal{F}_{3}$. To extract out the explicit temperature dependence from this term, we implement the procedure performed in Refs. [34,54]. The key issue is that although this term on the whole is convergent, (computing one at a time) the individual pieces are not. To circumvent this issue, we consider taking the Cauchy principal value of the integral (i.e., $\left.I_{3} \equiv F_{3}\right|_{\delta}$ ) and then we compute the difference between the original expression and this principal-valued integral. Formally, this is performed by taking,

$$
\frac{1}{x} \rightarrow \frac{1}{(x)_{\delta}}=\lim _{\delta \rightarrow 0} \frac{1}{x^{2}+\delta^{2}}
$$

The rest of the calculation is analogous to that presented in Ref. [34]. We indicate that it is helpful to perform the above $\delta$ integrals by using $\left(x^{2}+\delta^{2}\right)=(x+i \delta)(x-i \delta)$, and carefully performing the contour integration. The final result, after lengthy algebra, is (for the explicit-temperature dependence) a const. $+T$ scaling behavior (from both the principal value integral and the 'difference' terms), where the constant is the zero-temperature result.

\section{APPENDIX L: SELF-ENERGY AND RESISTIVITY}

The electrical resistivity is intimately related to the total relaxation lifetime (or scattering rate) of the conduction electron, which is taken to be

$$
\frac{1}{\tau_{\mathrm{tot}}}=\frac{1}{\tau_{0}}+\frac{1}{\tau_{K}},
$$

where $\tau_{\text {tot }}$ is the total conduction electron relaxation lifetime (or total time between scattering events), $\tau_{0}$ is the conduction electron lifetime in the absence of the Kondo interaction. $\tau_{K}$ is the relaxation lifetime correction arising from the Kondo interaction with the multipolar impurity. As is typical in the single impurity Kondo problem, the ordinary scattering rate in the absence of Kondo-scattering impurities $\left(1 / \tau_{0}\right)$ is taken 


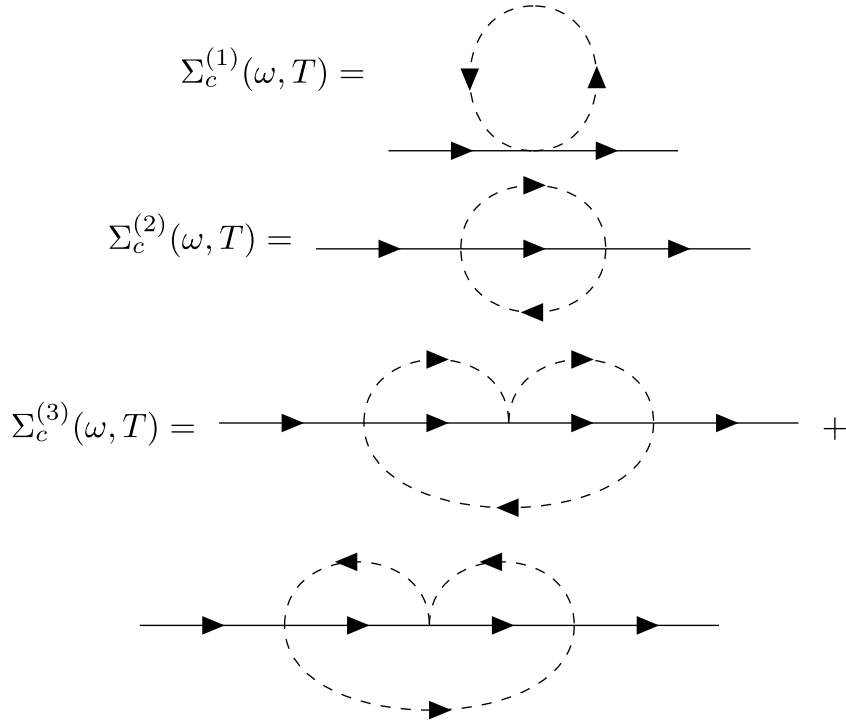

FIG. 9. Self-energy diagrams up to third order in perturbation theory.

to be much larger than the scattering rate due to the Kondo impurity due to the sheer scarcity of the number of multipolar impurities, i.e., $\tau_{0} \ll \tau_{K} . \tau_{K}$ arises via the Kondo exchange and is obtained from

$$
\frac{1}{\tau_{K}(\omega, T)}=-2 \operatorname{Im}\left(\Sigma_{c}(\omega, T)\right),
$$

where $\Sigma_{c}(\omega, T)$ is the conduction electron self-energy. Diagrammatically, the self-energy can be represented as sum of the Feynman graphs in Fig. 9.

The self-energy diagrams share a similarity with the free energy diagrams; this is not a coincidence as the free energy diagrams can be thought of as the self-energy diagrams but with the external conduction legs joined together. Just as in the free energy, the first order diagram vanishes due to taking $\operatorname{Tr}\left[S^{i}\right]=0$. We note that due to the matrix structure associated with the multipolar Kondo interaction, the selfenergy possesses a $10 \times 10$ matrix structure for the full model. We negate this difficulty by taking $\Sigma_{c}(\omega, T)$ in Eq. (L2) to be the sum of the eigenvalues of the self-energy matrix.

The dc conductivity can subsequently be obtained by insertion of the computed self-energy into the standard conductivity expression [55]

$$
\sigma(T)=\frac{n_{e} e^{2}}{m_{e}} \int_{0}^{\infty} d \omega \frac{\tau_{\text {tot }}(\omega)}{2 T \cosh ^{2}(\omega / 2 T)},
$$

where $n_{e}, e$, and $m_{e}$ are the conduction electrons' density, charge and mass, respectively, and $T$ is the temperature. We expand the total relaxation time in Eq. (L3) as $\tau_{\text {tot }}(\omega) \approx$ $\tau_{0}\left(1-\frac{\tau_{0}}{\tau_{K}(\omega)}\right)$, and invert Eq. (L3) expression to yield the resistivity $\rho \equiv \rho(T)-\rho_{0}$, where $\rho_{0}=\frac{m_{e}}{n_{e} e^{2}}$ is the ordinary resistivity and $\rho$ is the contribution arising from the multipolar Kondo exchange. Finally, we apply the RG procedure of taking the bare coupling constants to the running coupling constants to obtain the low-temperature behavior of the resistivity.
[1] B. Keimer, S. A. Kivelson, M. R. Norman, S. Uchida, and J. Zaanen, From quantum matter to high-temperature superconductivity in copper oxides, Nature (London) 518, 179 (2015).

[2] Q. Si, R. Yu, and E. Abrahams, High-temperature superconductivity in iron pnictides and chalcogenides, Nat. Rev. Mater. 1, 16017 (2016).

[3] A. Chubukov, Pairing Mechanism in Fe-based Superconductors, Annu. Rev. Condens. Matter Phys. 3, 57 (2012).

[4] P. Coleman, Theory perspective: Sces 2016, Philos. Mag. 97, 3527 (2017).

[5] A. C. Hewson, The Kondo Problem to Heavy Fermions, Cambridge Studies in Magnetism (Cambridge University Press, Cambridge, 1993).

[6] P. Phillips, Advanced Solid State Physics (Cambridge University Press, Cambridge, 2012).

[7] M. A. Ruderman and C. Kittel, Indirect exchange coupling of nuclear magnetic moments by conduction electrons, Phys. Rev. 96, 99 (1954).

[8] S. Doniach, The Kondo lattice and weak antiferromagnetism, Physica B+C 91, 231 (1977).

[9] I. Khait, P. Azaria, C. Hubig, U. Schollwöck, and A. Auerbach, Doped kondo chain, a heavy luttinger liquid, Proc. Natl. Acad. Sci. USA 115, 5140 (2018).

[10] S.-S. Lee, Recent developments in non-fermi liquid theory, Annu. Rev. Condens. Matter Phys. 9, 227 (2018).

[11] G. R. Stewart, Non-fermi-liquid behavior in $d$ - and $f$-electron metals, Rev. Mod. Phys. 73, 797 (2001).
[12] Y. Kuramoto, H. Kusunose, and A. Kiss, Multipole Orders and Fluctuations in Strongly Correlated Electron Systems, J. Phys. Soc. Jpn. 78, 072001 (2009).

[13] T. Onimaru and H. Kusunose, Exotic quadrupolar phenomena in non-kramers doublet systems - the cases of $\operatorname{PrT}_{2} \mathrm{Zn}_{20}(\mathrm{~T}=$ Ir, Rh) and $\operatorname{PrT}_{2} \mathrm{Al}_{20}(\mathrm{~T}=\mathrm{V}, \mathrm{Ti})$-, J. Phys. Soc. Jpn. 85, 082002 (2016).

[14] M. Tsujimoto, Y. Matsumoto, T. Tomita, A. Sakai, and S. Nakatsuji, Heavy-Fermion Superconductivity in the Quadrupole Ordered State of $\operatorname{PrV}_{2} \mathrm{Al}_{20}$, Phys. Rev. Lett. 113, 267001 (2014).

[15] T. J. Sato, S. Ibuka, Y. Nambu, T. Yamazaki, T. Hong, A. Sakai, and S. Nakatsuji, Ferroquadrupolar ordering in $\mathrm{PrTi}_{2}$ $\mathrm{Al}_{20}$, Phys. Rev. B 86, 184419 (2012).

[16] T. Onimaru, K. T. Matsumoto, Y. F. Inoue, K. Umeo, T. Sakakibara, Y. Karaki, M. Kubota, and T. Takabatake, Antiferroquadrupolar Ordering in a Pr-Based Superconductor PrIr ${ }_{2}$ $\mathrm{Zn}_{20}$, Phys. Rev. Lett. 106, 177001 (2011).

[17] A. Sakai, K. Kuga, and S. Nakatsuji, Superconductivity in the ferroquadrupolar state in the quadrupolar kondo lattice $\mathrm{PrTi}_{2}$ $\mathrm{Al}_{20}$, J. Phys. Soc. Jpn. 81, 083702 (2012).

[18] K. Matsubayashi, T. Tanaka, A. Sakai, S. Nakatsuji, Y. Kubo, and Y. Uwatoko, Pressure-Induced Heavy Fermion Superconductivity in the Nonmagnetic Quadrupolar System $\operatorname{PrTi}_{2} \mathrm{Al}_{20}$, Phys. Rev. Lett. 109, 187004 (2012).

[19] S. C. Riggs, M. C. Shapiro, A. V Maharaj, S. Raghu, E. D. Bauer, R. E. Baumbach, P. Giraldo-Gallo, M. Wartenbe, and 
I. R. Fisher, Evidence for a nematic component to the hiddenorder parameter in $\mathrm{URu}_{2} \mathrm{Si}_{2}$ from differential elastoresistance measurements, Nat. Commun. 6, 6425 (2015).

[20] E. W. Rosenberg, J.-H. Chu, J. P. C. Ruff, A. T. Hristov, and I. R. Fisher, Divergence of the quadrupole-strain susceptibility of the electronic nematic system $\mathrm{YbRu}_{2} \mathrm{Ge}_{2}$, Proc. Natl. Acad. Sci. USA 116, 7232 (2019).

[21] F. Freyer, J. Attig, SungBin Lee, A. Paramekanti, S. Trebst, and Y. B. Kim, Two-stage multipolar ordering in $\operatorname{PrT}_{2} \mathrm{Al}_{20}$ Kondo materials, Phys. Rev. B 97, 115111 (2018).

[22] K. Hattori and H. Tsunetsugu, Antiferro quadrupole orders in non-kramers doublet systems, J. Phys. Soc. Jpn. 83, 034709 (2014).

[23] SungBin Lee, S. Trebst, Y. B. Kim, and A. Paramekanti, Landau theory of multipolar orders in $\operatorname{Pr}(\mathrm{Y})_{2} \mathrm{X}_{20}$ Kondo materials ( $\mathrm{Y}=$ Ti, V, Rh, Ir; X = Al, Zn), Phys. Rev. B 98, 134447 (2018).

[24] A. S. Patri, A. Sakai, SungBin Lee, A. Paramekanti, S. Nakatsuji, and Y. B. Kim, Unveiling hidden orders: Magnetostriction as a probe of multipolar-ordered states, Nat. Commun. 10, 4092 (2019).

[25] J. A. Mydosh and P. M. Oppeneer, Colloquium: Hidden order, superconductivity, and magnetism: The unsolved case of $\mathrm{uru}_{2} \mathrm{si}_{2}$, Rev. Mod. Phys. 83, 1301 (2011).

[26] P. Santini, S. Carretta, G. Amoretti, R. Caciuffo, N. Magnani, and G. H. Lander, Multipolar interactions in $f$-electron systems: The paradigm of actinide dioxides, Rev. Mod. Phys. 81, 807 (2009).

[27] D. L. Cox, Quadrupolar Kondo Effect in Uranium HeavyElectron Materials? Phys. Rev. Lett. 59, 1240 (1987).

[28] D. L. Cox, The quadrupolar kondo effect: A new mechanism for heavy electrons, J. Magn. Magn. Mater. 76-77, 53 (1988).

[29] A. Sakai and S. Nakatsuji, Kondo effects and multipolar order in the cubic $\operatorname{PrTr}_{2} \mathrm{Al}_{20}(\mathrm{Tr}=\mathrm{Ti}, \mathrm{V})$, J. Phys. Soc. Jpn. 80, 063701 (2011).

[30] T. Onimaru, K. Izawa, K. T. Matsumoto, T. Yoshida, Y. Machida, T. Ikeura, K. Wakiya, K. Umeo, S. Kittaka, K. Araki, T. Sakakibara, and T. Takabatake, Quadrupole-driven nonFermi-liquid and magnetic-field-induced heavy fermion states in a non-Kramers doublet system, Phys. Rev. B 94, 075134 (2016).

[31] Y. Shimura, M. Tsujimoto, B. Zeng, L. Balicas, A. Sakai, and S. Nakatsuji, Field-induced quadrupolar quantum criticality in $\mathrm{PrV}_{2} \mathrm{Al}_{20}$, Phys. Rev. B 91 241102(R) (2015).

[32] S. Nagashima, T. Nishiwaki, A. Otani, M. Sakoda, E. Matsuoka, H. Harima, and H. Sugawara, De Haas-van Alphen Effect in $\mathrm{RTi}_{2} \mathrm{Al}_{20}(\mathrm{R}=\mathrm{La}, \mathrm{Pr}$, and Sm), JPS Conf. Proc. 3, 011019 (2014).

[33] Ph. Nozières and A. Blandin, Kondo effect in real metals, J. Phys. (France) 41, 193 (1980).

[34] J. Gan, On the multichannel Kondo model, J. Phys.: Condens. Matter 6, 4547 (1994).

[35] N. Andrei and C. Destri, Solution of the Multichannel Kondo Problem, Phys. Rev. Lett. 52, 364 (1984).

[36] A. W. W. Ludwig and I. Affleck, Exact, Asymptotic, ThreeDimensional, Space- and Time-Dependent, Green's Functions in the Multichannel Kondo Effect, Phys. Rev. Lett. 67, 3160 (1991).

[37] I. Affleck, Conformal field theory approach to the kondo effect, Acta Phys. Pol. B 26, 1869 (1995).

[38] P. Gegenwart, Q. Si, and F. Steglich, Quantum criticality in heavy-fermion metals, Nat. Phys. 4, 186 (2008).

[39] I. Affleck, Quantum impurity problems in condensed matter physics, in Exact Methods in Low-dimensional Statistical Physics and Quantum Computing, Vol. 89 (Oxford University Press, 2008), pp. 3-65.

[40] I. Affleck and A. W. W. Ludwig, Exact conformal-fieldtheory results on the multichannel kondo effect: Single-fermion green's function, self-energy, and resistivity, Phys. Rev. B 48, 7297 (1993).

[41] A. W.W. Ludwig, Exact results on the multi-channel kondo effect from conformal field theory, Physica B: Condensed Matter 199-200, 406 (1994).

[42] N. Andrei, K. Furuya, and J. H. Lowenstein, Solution of the kondo problem, Rev. Mod. Phys. 55, 331 (1983).

[43] A. M. Tsvelick and P. B. Wiegmann, Exact solution of the multichannel kondo problem, scaling, and integrability, J. Stat. Phys. 38, 125 (1985).

[44] A. M. Tsvelick and P. B. Wiegmann, Solution of then-channel kondo problem (scaling and integrability), Z. Phys. B 54, 201 (1984).

[45] H.-H. Lai, E. M. Nica, W.-J. Hu, S.-S. Gong, S. Paschen, and Q. Si, Kondo destruction and multipolar order- implications for heavy fermion quantum criticality, arXiv:1807.09258 [condmat.str-el].

[46] V. Madhavan, W. Chen, T. Jamneala, M. F. Crommie, and N. S. Wingreen, Tunneling into a single magnetic atom: Spectroscopic evidence of the kondo resonance, Science 280, 567 (1998).

[47] J. Li, W.-D. Schneider, R. Berndt, and B. Delley, Kondo Scattering Observed at A Single Magnetic Impurity, Phys. Rev. Lett. 80, 2893 (1998).

[48] T.-S. Kim and D. L. Cox, One-, two-, and three-channel kondo effects for a model $\mathrm{ce}^{3+}$ impurity in a metal, Phys. Rev. B 55, 12594 (1997).

[49] H. B. Pang and D. L. Cox, Stability of the fixed point of the two-channel Kondo Hamiltonian, Phys. Rev. B 44, 9454 (1991).

[50] R. Ul Haq, S. S. Bharadwaj, and T. A. Wani, An explicit method for schrieffer-wolff transformation, arXiv:1901.08617 [cond-mat.str-el].

[51] D. L. Cox and A. Zawadowski, Exotic kondo effects in metals: Magnetic ions in a crystalline electric field and tunneling centres, Adv. Phys. 47, 599 (1998).

[52] P. W. Anderson, A poor man's derivation of scaling laws for the kondo problem, J. Phys. C: Solid State Phys. 3, 2436 (1970).

[53] S. Weinberg, The Quantum Theory of Fields (Cambridge University Press, Cambridge, 1996), Vol. 2, Chap. 18.

[54] J. Kondo, Free-energy shift of conduction electrons due to the s-d exchange interaction, Prog. Theor. Phys. 40, 683 (1968).

[55] A. A. Abrikosov, Electron scattering on magnetic impurities in metals and anomalous resistivity effects, Physics Physique Fizika 2, 5 (1965). 